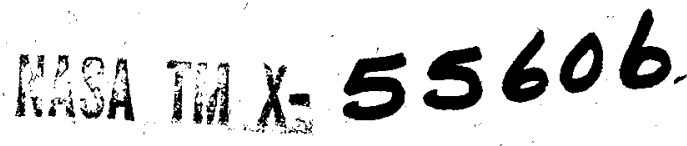

\section{LONGITUDE DEPENDENCE OF GEOMAGNETICALLY TRAPPED ELECTRONS}

GPO PRICE

CFSTI PRICE(S)

Microfiche (MF)

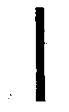

Hard copy $(\mathrm{HC})$

ff 653 July 85
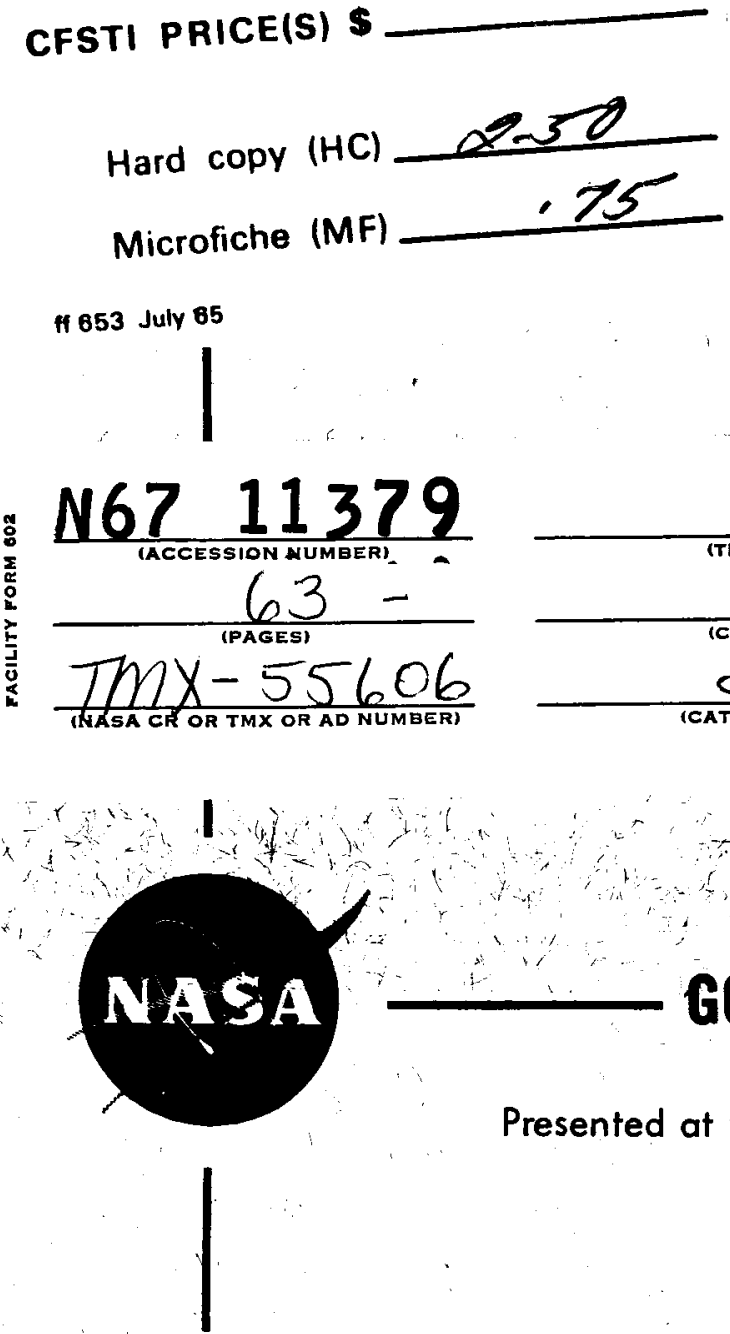

BY

J. G. ROEDERER

J. A. WELCH

J. V. HEROD

\section{AUGUST 1966}

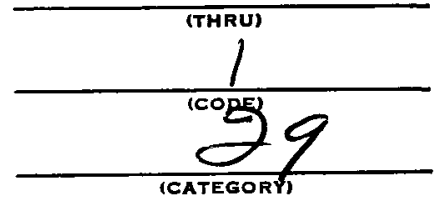

GODDARD SPACE FLIGHT CENTER

\section{GREENBELT, MARYLAND}

Presented at the Inter-Union Symposium on Solar-Terrestrial Physics, Belgrade August 28 - September 2, 1966 


\section{LONGITUDE DEPENDENCE OF GEOMAGNETICALLY}

\section{TRAPPED ELECTRONS}

PART I

by

Juan G. Roederer *

Goddard Space Flight Center

Greenbelt, Maryland

and

Jasper A. Welch ${ }^{\star \star}$

Air Force Systems Command

Los Angeles, California

*National Academy of Sciences - National Research Council, Senior Post Doctoral Research Associate.

Present address: Centro Nacional de Radiacion Cosmica Peru 272, Buenos Aires, Argentina $\star \star$ Lieutenant Colonel, United States Air Force 


$$
\text { W67-11379 ABSTRACT }
$$

A general Fokker-Planck equation is deduced, which describes the distribution of geomagnetically trapped electrons as a function of longitude, time, energy and mirror point field intensity. A special variable for the longitudinal position of a particle is introduced. The coefficients representing longitudinal drift, ionization loss and multiple Coulomb scattering in the Fokker-Planck equation are derived. The physical interpretation of this equation is analyzed for several special cases. It is found that the usual procedure of averaging over longitude in order to obtain a longitude - independent description is not valid for electrons mirroring at low altitudes in the South American Anomaly. The correct procedure is discussed. It is further concluded, that the region East of the Anomaly, initially depleted by precipitation, is replenished by electrons whose mirror points were situated in a narrow "window" in B, before passing through the Anomaly. A very limited extension of the atmosphere in the Anomaly should control this mechanism of replenishment by Coulomb scattering. A small diurnal effect for the low altitude electron flux is predicted, determined by the diurnal variation of the atmosphere in the Anomaly.

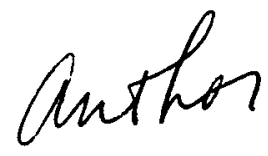




\section{LONGITUDE DEPENDENCE OF GEOMAGNETICALLY}

\section{TRAPPED ELECTRONS}

PART I

\section{INTRODUCTION}

Time has come to look with more detail into the latitude dependence of geomagnetically trapped particle fluxes from the theoretical point of view. Until rccently, experimental information on this subject was very scarce. Only after the discovery of a region over the East coast of South America and the adjacent Atlantic Ocean, with enhanced fluxes of trapped radiation at a given altitude (Yoshida et al. ${ }^{1}$, Vernov et al. ${ }^{2}$ ), attention got more and more focussed on the experimental analysis of particle precipitation in the Anomaly, and the mechanism of subsequent replenishment of the depleted shell regions. In particular, Imhof and Smith ${ }^{3}$, Paulikas and Freden ${ }^{4}$ and Mihalov et al. ${ }^{5}$ have made a careful study of artifically injected electron fluxes, measured by various satellites. On the other hand, Freden and Paulikas ${ }^{6}$ and Heckman and Nakano ${ }^{7}$ have analyzed proton fluxes at low altitudes in the Anomaly. Evidence for Bremsstrahlung $\mathrm{X}$-rays from electrons precipitating into the Anomaly was found by Ghielmetti, et. al. ${ }^{8}$. A thorough study of the longitude dependence of electron fluxes was published by Williams and Kohl ${ }^{9}$. It is therefore desirable to set up a theoretical description of the longitudinal behavior of trapped particles, and to test by comparison with experimental data, the various assumptions made about interaction processes governing particle diffusion.

Theoretical description of electron trapping, diffusion and precipitation was so far done only for configurations averaged over all longitudes $10,11,12,13,14$. 
In these papers, a time-dependent Fokker-Planck equation was set up for the electron distribution function and used to follow the evolution in time of a given, initial electron flux.

In order to study the longitude dependence of the electron distribution on a given magnetic shell, it is necessary to derive a more general diffusion equation which contains an additional variable related to longitude. In this equation the coefficients, which depend upon the atmosphere, will be a function of longitude and local time of day in the Anomaly. One important by-product of the equation derivation will be the identification of an appropriate variable by which to describe the longitudinal dependence. We will call this variable $X$. It will be used, together with the particle energy $\mathbf{E}$, the scalar magnetic field $B$ at the mirror point, and the well known Mcllwain shell parameter $L, 15$ to describe the space in which particle densities change with time, $t$.

The main purpose of part I is to set up such a general equation, to discuss its physical meaning, to compare it with the previously used longitude-averaged equation, and to draw some general, qualitative conclusions. The second part will deal with atmosphere-field configurations to be used in this longitude dependent description, and results of a numerical integration of the general equation will be presented.

Before setting up our equation, let us picture the problem in general terms. Consider the familiar B-L space, ${ }^{15}$ in which trapped radiation fluxes are usually described (Fig. 1). It can be shown that electrons whose mirror points are below $100 \mathrm{~km}$ cannot remain trapped for more than a few bounces. Thus, it is qualitatively useful to consider the $100 \mathrm{~km}$ level as the location of a sink. We shall return to this point in more detail later. At a given longitude $X$ the $100 \mathrm{~km}$ 


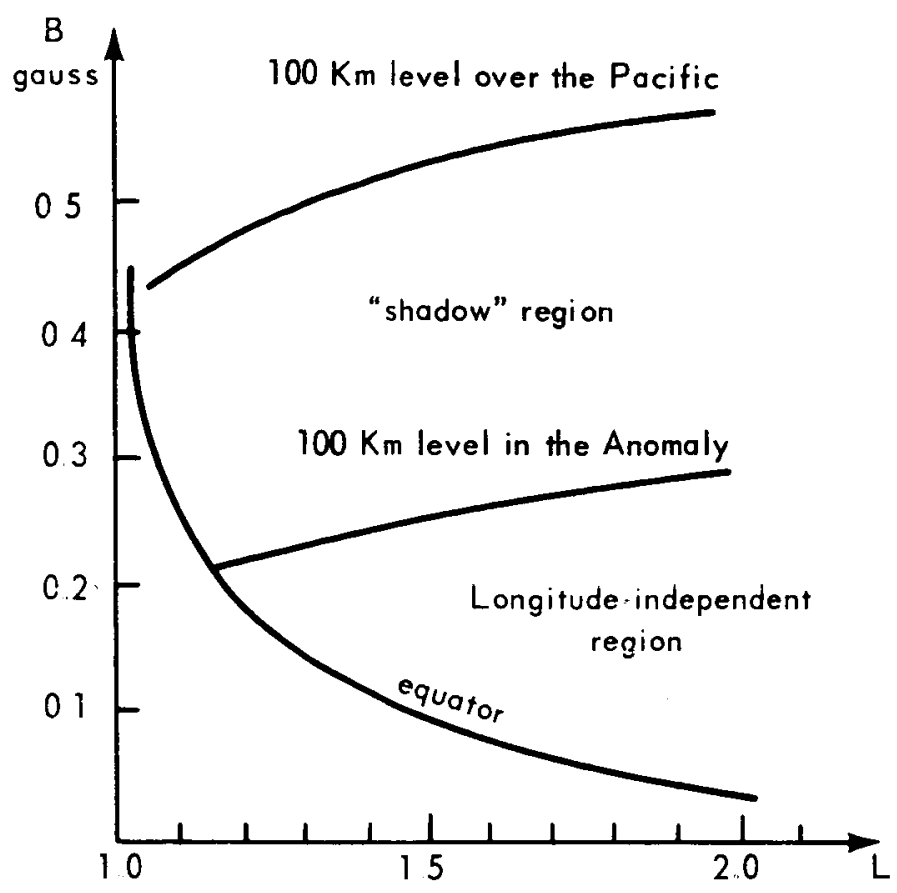

Figure 1-The "windshield wiper" effect in B-L space.

level can be displayed as a locus $B_{c}(L, X)$ in B-L space. Essentially no electrons will be found with mirror points above this curve, that is, for mirror point fields larger than $B_{c}(L, X)$. We have plotted two extreme loci of the $100 \mathrm{~km}$ level, corresponding to a longitude (that is, a value of $\mathrm{X}$ ) right in the "center" of the South American Anomaly, and a longitude over the Pacific Ocean, respectively. From Fig. 1, one can see that for particles of a given energy, the $100 \mathrm{~km}$ curve, $B_{c}(1, X)$, "oscillates" with longitudinal drift frequency between these two extreme positions. As $B_{c}(L, X)$ lowers from its maximum, or "Pacific," position, the region in B-L space between the two extreme positions, called the "shadow region," is wiped clean of particles. As $B_{c}(L, X)$ rises from its minimum, or "Anomaly," position, the opportunity exists for atmospheric scattering, or any other nonadiabatic process, to repopulate this shadow region. This wiping clean and repopulation has been likened to the action of a windshield wiper. 
Satellite observations do, in fact, find electrons in the shadow region 3,9 . This observation means that particles have enough non-adiabatic interaction during one drift around the earth to diffuse into the wiped-out or "shadow" region. Our main goal is to study this process of fast replenishment and to determine to what extent it can be explained by the process of interaction of electrons with the atmosphere through Coulomb scattering and energy loss.

\section{REPRESENTATION OF THE ELECTRON DISTRIBUTION}

\section{AS A FUNCTION OF LONGITUDE}

We shall describe the trapped electrons by the distribution function used by Welch, Kaufmann and Hess ${ }^{11}$. We shall denote by

$$
\delta \mathrm{N}=\mathrm{U}(\mathrm{I}, \mathrm{B}, \mathrm{X}, \mathrm{E}, \mathrm{t}) \delta \Phi \delta \mathrm{B} \delta \mathrm{E}
$$

the number of electrons contained at the time $t$ in a tube of field lines of magnetic flux $\delta \Phi=\mathrm{B}_{0} \delta \mathrm{A}$, situated at a longitude characterized by the (not yet defined) parameter $X$, with mirror points between $B$ and $B+\delta B$, and with energies between $\mathrm{E}$ and $\mathrm{E}+\delta \mathrm{E}$ (See Fig. 2). In this paper, the mirror point field will always be denoted by $B$ without suffix. The shell on which these electrons drift is characterized by the two adiabatic invariants $\mu$ and ${ }^{16}$, which for static fields and in absence of an electric field reduce to the field-geometric invariants

$$
\begin{gathered}
B_{(m i r r o r)}=\text { const } . \\
I=\int_{m}^{m^{\prime}} \sqrt{1-\frac{B(s)}{B}} d s=\text { const. }
\end{gathered}
$$


respectively. For the time being, we shall make no limiting assumption regarding azimuthal symmetry; this means that particles initially on one and the same field line may not at all stay on a common shell during their drift in longitude ${ }^{17,18}$.

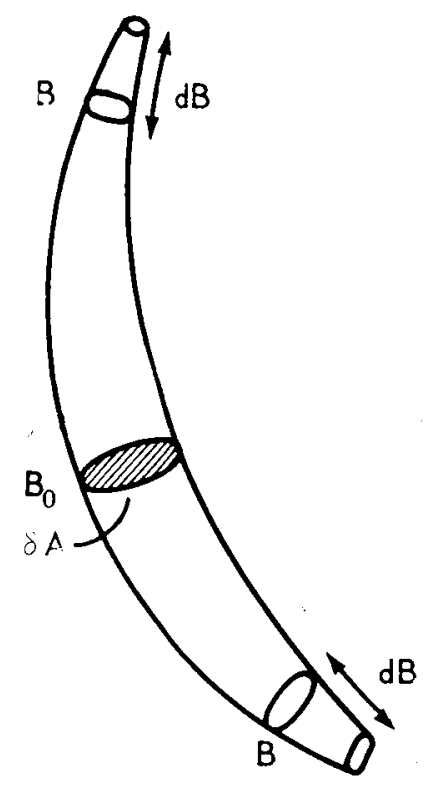

Figure 2

The distribution $U$ defined in (1) is in general not directly measurable. Relations with other particle distribution quantities are given in ${ }^{11}$. In particular, $U$ is related to the directional, differential flux at the equator $\mathrm{j}_{0}=\mathrm{j}_{0}\left(\mathrm{I}, \alpha_{0}, \mathrm{X}, \mathrm{E}, \mathrm{t}\right)$ by the expression

$$
\mathrm{U}=\frac{2 \pi}{\mathrm{B}^{2}} \mathrm{j}_{0} \tau_{\mathrm{b}}
$$

$a_{0}=\arcsin \sqrt{B_{0} / B}$ is the equatorial pitch angle of a particle mirroring at $B ; \tau_{b}$ is the bounce half-period (time to travel from one mirror point to its conjugate). In an azimuthally asymmetric field, the pitch angle $\alpha_{0}$ of a given particle is in general a function of longitude $a_{0}=a_{0}(B, X)$ (in these fields, the field intensity 
$B_{0}$ at the equatorial ring of a shell varies with longitude). This is why we prefer to use the distribution function $U$, which is represented in terms of the longitudinally invariant variable B.

Let us now consider electrons of a given energy, mirroring at the same Bvalue, trapped between two neighboring shells labeled with the values of the second invariants, $I$ and $I+\delta I$, respectively. $I+\delta I$ is the value of the second invariant, taken along a line of force belonging to the outer shell, between mirror points with the same field intensity B. We shall call $\nabla_{0} I=\vec{n} \delta I / \delta$ y the gradient of $I$ at the equatorial point of a magnetic shell (Figs. 3,4). Northrop and Teller ${ }^{16}$ have shown that the equatorial drift velocity, averaged over one bounce, is given by

$$
\vec{u}_{0}=\frac{\mathrm{mv}}{\mathrm{eB}_{0}^{2} \tau_{\mathrm{b}}} \vec{\nabla}_{0} \mathrm{I} \times \overrightarrow{\mathrm{B}}=\frac{\mathrm{p}}{\mathrm{eB}} \frac{\nabla_{0} \mathrm{I}}{\tau_{\mathrm{b}}} \overrightarrow{\mathrm{b}}
$$

where $p$ is the particle's momentum, e the charge ( $<0$ for electrons) and $\tau_{b}$ is the half-period of bouncing. $\quad B_{0}$ is the equatorial $B$-value. For the different vectors, see Fig. 4. Introducing $\mathrm{S}_{\mathrm{b}}$, rectified path of the particle between mirror points, we can write

$$
\vec{u}_{0}=\beta^{2} \gamma \frac{m_{0} c^{2}}{e B_{0}} \frac{\nabla_{0} I}{S_{b}} \vec{b}
$$

$\beta$ and $\gamma$ are the usual relativistic factors. Notice the following useful general expression for the path $S_{b}$, which can be obtained straight-forward by taking the derivative of $(2 \mathrm{~b})$ with respect to the mirror point field $\mathrm{B}$, along a given field line:

$$
S_{b}=I+2 B \frac{\partial I}{\partial B}
$$




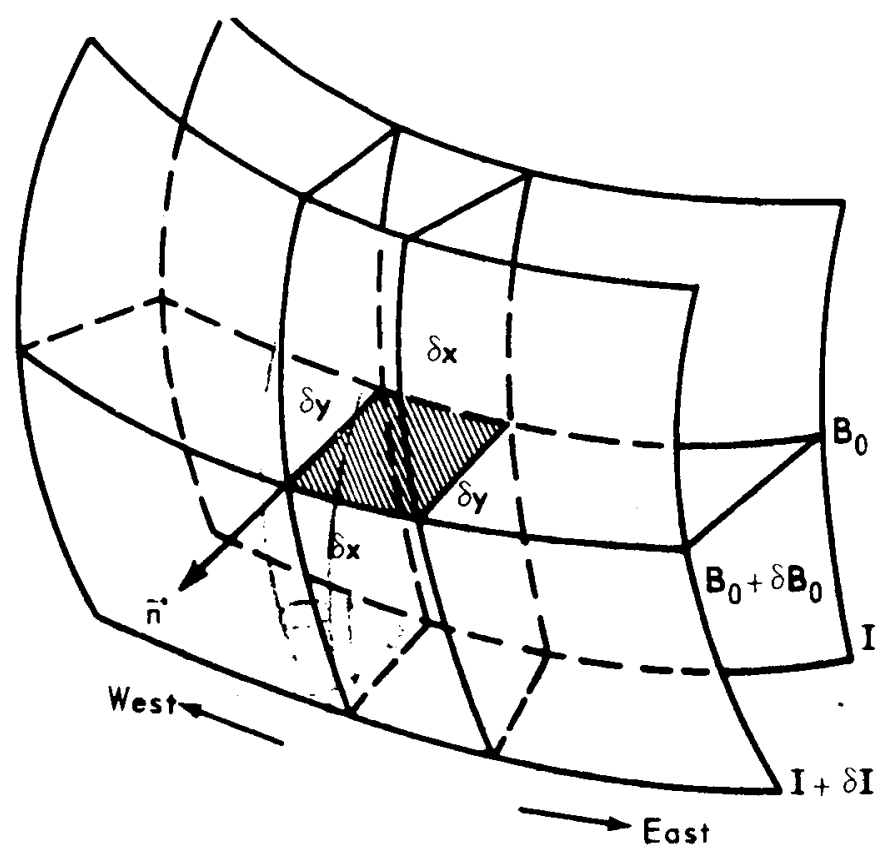

Figure 3-Shell geometry and elementary flux tube

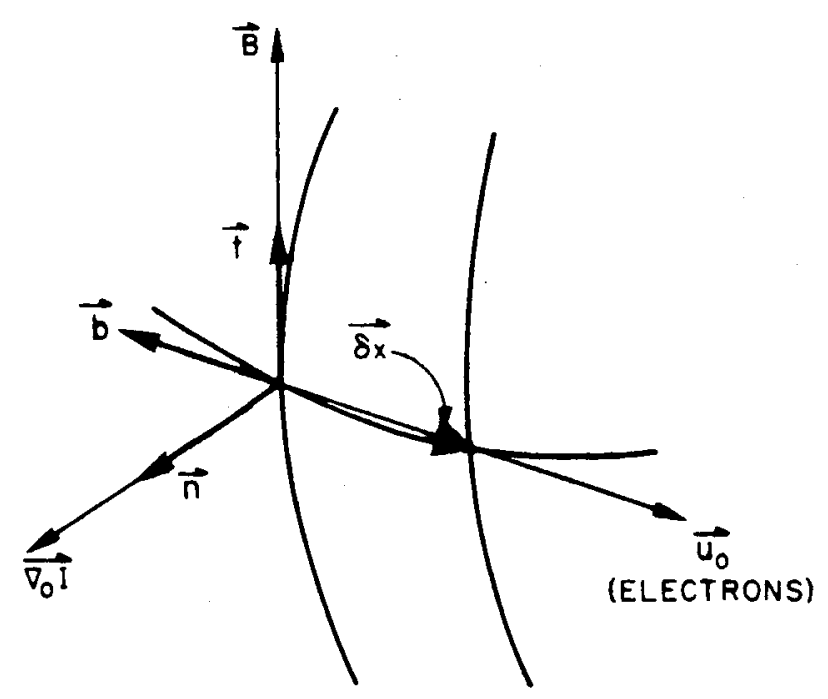

Figure 4-Unit Vectors', mean equatorial drift velocity and gradient of 1 
We denote with $\delta \mathbf{x}=u_{0} \delta \mathrm{t}$ the distance between equatorial points of two neighboring field lines of a given shell; $\delta \mathbf{x}$ is then the element of arc of the shell's equatorial $\mathrm{B}_{0}-\mathrm{ring} ; \mathrm{x}=\int \mathrm{dx}$ is the total arc length along the equator from a given initial point and can be used as a label to locate field lines on a given shell. Evidently

$$
\mathbf{x}=\int_{\varphi_{0}}^{\varphi} \frac{\partial \mathbf{x}}{\partial \varphi} \mathrm{d} \varphi
$$

where $\partial \mathbf{x} / \partial \varphi$ is a field-geometric factor $(\partial \mathbf{x} / \partial \varphi=2 \pi R L / 360$ for a centered dipole), and $\varphi$ the geographic longitude of the equatorial point. Notice that for the angular equatorial drift velocity,

$$
\dot{\varphi}_{0}=\frac{1}{\partial \mathbf{x} / \partial \varphi} u_{0}
$$

Let us take a tube of lines of force of equatorial cross section $\delta \mathbf{x} \delta \mathbf{y}$ and magnetic flux $B_{0} \delta x \delta y \quad$ (Fig. 3). By the definition of $U,(1)$, the number of electrons in this flux tube, which mirror between $B$ and $B+\delta B$ and with kinetic energy between $\mathbf{E}$ and $\mathbf{E}+\delta \mathrm{E}$, is given by

$$
\delta \mathbf{N}=\mathrm{UB}_{0} \delta \mathbf{x} \delta \mathbf{y} \delta \mathbf{B} \delta \mathbf{E}
$$

We now want to follow the history of these particles, as a function of time. Before setting up the Boltzmann equation governing the distribution $\mathrm{U}$, we have to introduce a convenient variable for the longitude, i.e., a convenient label for the field line of a given magnetic shell, around which a particle is instantaneously spiralling. We cannot take $\varphi$ or $x$ (5) as suitable variables, because $\mathrm{U}$ is a distribution in flux, not in $\varphi$ or $\mathbf{x}$. And in the flux expression $\mathrm{B}_{0} \delta \mathbf{x} \delta \mathbf{y}$ 
intervening in (7), not only $\delta x$, but also $\delta y$ is a function of longitude, for the general geomagnetic field.

In order to find the correct longitudinal variable, we have to transform the flux into an expression $\delta \Phi=\delta \mathbf{X} \delta \mathrm{Y}$, in which $\delta \mathrm{Y}$ - still related to the radial increment $\delta \mathrm{y}-$ is now longitude-independent, so that it may be ignored when following the particles during their longitudinal drift. In that case, $\mathrm{X}$ would be the correct longitudinal variable, its differential $\delta \mathbf{X}$ containing complete information about the longitude dependence of the flux $\delta \Phi$ of a tube filled with particles, as they drift around the earth. It is easy to realize that, in our notation, $\mathrm{X}$ and $\mathrm{Y}$ must be proportional to a pair of " $\alpha, \beta$ " coordinates ${ }^{19}$.

In order to find $\mathrm{X}$ and $\mathrm{Y}$, let us write the obvious relation (Figs. 3 and 4 )

$$
\delta \mathbf{y}=\frac{\delta \mathbf{I}}{\nabla_{0} \mathbf{I}}
$$

Introducing this into the expression for the flux element, we have:

$$
\delta \Phi=\mathrm{B}_{0} \delta \mathbf{x} \delta \mathbf{y}=\mathrm{B}_{0} \delta \mathbf{x} \frac{\delta \mathbf{I}}{\nabla_{0} \mathrm{I}}
$$

A possible pair of coordinates is therefore

$$
\begin{gathered}
X=\int B_{0} \frac{d x}{\nabla_{0} I} \\
Y=I
\end{gathered}
$$

The integral (8a) is to be performed along the equatorial ring of a given I, Bshell, starting at a fixed reference longitude. We must emphasize again that $\mathrm{B}_{0}$ is in general longitude dependent, and therefore cannot be taken out of the integral (8a). Notice further that our system $X, Y$ is independent of the particle's energy, depending only on the field geometry. 
Using (8a) and (8b), the flux element becomes

$$
\delta \Phi=\mathrm{B}_{0} \delta \mathbf{x} \delta \mathrm{y}=\delta \mathbf{X} \delta \mathrm{Y}
$$

where $\delta Y(=\delta I)$ is now independent of longitude and can be left out in the entire following discussion. With this new variable $X$, the longitudinal drift velocity becomes, according to (8a) and (4):

$$
\dot{\xi}=\frac{\mathrm{dX}}{\mathrm{dt}}=\mathrm{u}_{0} \frac{\mathrm{dX}}{\mathrm{dx}}=\mathrm{u}_{0} \frac{\mathrm{B}_{0}}{\nabla_{0} I}=\frac{\mathrm{p}}{\mathrm{e} \tau_{\mathrm{b}}}=\frac{\mathrm{m}_{0}}{\mathrm{e}} \beta^{2} \gamma \frac{1}{\mathrm{~s}_{\mathrm{b}}}
$$

Remember that $S_{b}$ is in general longitude dependent. Notice the following relation, taking into account (5) and (6):

$$
\frac{\mathrm{e}}{\mathrm{p}} \tau_{\mathrm{b}} \mathrm{dX}=\frac{\mathrm{d} \mathrm{x}}{\mathrm{u}_{0}}=\frac{\mathrm{d} \varphi}{\dot{\varphi}_{0}}
$$

\section{THE GENERAL FOKKER-PLANCK EQUATION FOR TRAPPED ELEC- TRON DIFFUSION}

We are now in condition to set up the general equation governing the distribution of trapped electrons. These electrons will undergo displacements in B, $\mathrm{E}$ and longitude, caused by three types of mutually independent interactions:

(1a) A change in I, coupled to a change in B due to the stochastic process of pitch angle scattering (multiple Coulomb scattering in the atmosphere, interactions with traveling electromagnetic waves, etc.);

(1b) A change in I, B and E due to the stochastic process of radial diffusion across shells (violation of the third adiabatic invariant during geomagnetic perturbations, etc.);

(2) A change in kinetic energy $\mathrm{E}$, due to ionization loss in the atmosphere, considered here as a non-stochastic process (i.e., neglecting straggling); 
(3) A change in longitude due to interaction with the static magnetic field (longitudinal drift), again a non-stochastic process.

All these processes are physically in dependent of each other, although all intervening parameters are in general functions of the three variables $\mathrm{B}, \mathrm{X}$ and $\mathrm{E}$. Let us relate the distribution of electrons as it appears in (20), with the distribution of electrons at a slightly earlier time $t-\Delta t$, at a different longitudinal position. For the time being, we shall forget the non-stochastic character of $\mathbf{E}$ and $\mathrm{X}$, and treat all variables as if they were of the same, stochastic nature. We write:

$$
\begin{aligned}
& \mathrm{U}(\mathbf{I}, \mathbf{B}, \mathbf{X}, \mathbf{E}, \mathbf{t})= \\
& \iiint \int \mathrm{U}(\mathbf{I}-\eta, \mathbf{B}-\beta, \mathbf{X}-\xi, \mathbf{E}-\epsilon, \mathbf{t}-\Delta \mathbf{t}) \Pi(\mathbf{I}-\eta, \mathbf{B}-\beta, \mathbf{X}-\xi, \mathbf{E}-\epsilon, \\
& \quad \eta, \beta, \xi, \epsilon, \Delta \mathbf{t}) \mathrm{d} \eta \mathrm{d} \beta \mathrm{d} \xi \mathrm{d} \epsilon+\mathrm{Q}(\mathbf{I}, \mathbf{B}, \mathbf{X}, \mathbf{E}, \mathbf{t}) \Delta \mathbf{t}
\end{aligned}
$$

In this relation, the distribution of electrons which at the time $t$ are at a position $X$, is linked to the distribution of those electrons which at an earlier time $t-\Delta t$ were at $X-\xi$, and which happened to diffuse, scatter, slow down and drift the right amount in the interval $\Delta t$, in order to become part of the population described by the left hand of (11).

$\Pi \mathrm{d} \eta \mathrm{d} \beta \mathrm{d} \xi \mathrm{d} \epsilon$ is the a priori probability that these electrons have undergone just the right changes in I, mirror point field, longitudinal position and energy, in the time interval $\Delta t$.

The source term $Q \Delta t$ represents the contributions of electrons added by injection to the original bunch of particles, during $\Delta t$. 
Expanding all intervening functions in Taylor series in $\eta, \beta, \xi, \epsilon$ and $\Delta \mathfrak{t}$, we obtain:

$$
\begin{aligned}
& \frac{\partial \mathbf{u}}{\partial \mathbf{t}}+\frac{\partial}{\partial \mathbf{X}}(\mathrm{U}\langle\xi\rangle)=\frac{\partial}{\partial \mathrm{I}}(\mathrm{U}\langle\eta\rangle)+\frac{1}{2} \frac{\partial^{2}}{\partial \mathbf{I}^{2}}\left(\mathrm{U}\left\langle\eta^{2}\right\rangle\right)- \\
& -\frac{\partial}{\partial \mathrm{B}}(\mathrm{U}\langle\beta\rangle)+\frac{1}{2} \frac{\partial^{2}}{\partial \beta^{2}}\left(\mathrm{U}\left\langle\beta^{2}\right\rangle\right)-\frac{\partial}{\partial \mathrm{F}}(\mathrm{U}\langle\epsilon\rangle)+\mathrm{Q}+
\end{aligned}
$$

+ cross terms and higher order terms

The brackets stand for the average per unit time of the enclosed variables:

$$
\langle\cdots\rangle=\frac{1}{\Delta \mathrm{t}} \iiint \int \cdots \cdot \Pi \mathrm{d} \eta \mathrm{d} \beta \mathrm{d} \xi \mathrm{d} \epsilon
$$

We can now re-instate the non-stochastic character to $X$, by taking $\Pi$ as a delta function in longitude:

$-\Pi(\mathrm{I}, \mathrm{B}, \mathrm{X}, \mathrm{E}, \eta, \beta, \xi, \epsilon, \Delta \mathrm{t})=\Pi_{0}(\mathrm{I}, \mathrm{B}, \mathrm{X}, \mathrm{E}, \eta, \beta, \epsilon, \Delta \mathrm{t}) \delta(\xi-\dot{\xi} \Delta \mathrm{t})$

$\dot{\xi}$ is given by (9). $\Pi_{0}$ is the probability for a change in $I, B$ and $E$ due to radial and pitch angle diffusion, in the interval $\Delta t$. It is conveniently expressed by

$$
\Pi_{0}(\mathrm{I}, \mathrm{B}, \mathrm{X}, \mathrm{E}, \eta, \beta, \epsilon, \Delta \mathrm{t})=\frac{\mathrm{P}(\mathrm{I}, \mathrm{B}, \mathrm{X}, \mathrm{E}, \eta, \beta, \epsilon,)}{\tau_{\mathrm{b}}(\mathrm{I}, \mathrm{B}, \mathrm{X}, \mathrm{E})} \Delta \mathrm{t}
$$

where $P$ is the scattering probability per one half-bounce. In all this we have implicitly assumed that scattering effects are extremely small during one half-bounce of the particle. In other words, we suppose that $\mathbf{P} \ll 1$ for $\eta, \beta, \epsilon, 10$ and approximately $\mathbf{P} \cong 1$ for $\eta, \beta, \epsilon,=0$. Equation (11) finally becomes:

$$
\begin{aligned}
& \frac{\partial \mathrm{U}}{\partial \mathrm{t}}+\frac{\partial}{\partial \mathrm{X}}(\mathrm{U} \dot{\xi})=-\frac{\partial}{\partial \mathrm{I}}(\mathrm{U}\langle\eta\rangle)+\frac{1}{2} \frac{\partial^{2}}{\partial \mathrm{I}^{2}}\left(\mathrm{U}\left\langle\eta^{2}\right\rangle\right)- \\
& -\frac{\partial}{\partial \mathrm{B}}(\mathrm{U}\langle\beta\rangle)+\frac{1}{2} \frac{\partial^{2}}{\partial \mathrm{B}^{2}}\left(\mathrm{U}\left\langle\beta^{2}\right\rangle\right)-\frac{\partial}{\partial \mathrm{E}}(\mathrm{U}\langle\epsilon\rangle)+\mathrm{Q}+ \\
& + \text { cross terms and higher order terms }
\end{aligned}
$$


This is the most general equation of diffusion for trapped particles, applicable for an arbitrary trapping field geometry and for arbitrary radial and pitch angle diffusion processes. All coefficients \langle\rangle (average changes per unit time), can be expressed as average changes per half-bounce, in the form:

$$
\langle\cdots\rangle=\frac{1}{\tau_{b}}\{\cdots \cdot\}
$$

whore

$$
\{\cdots \cdot\}=\iiint \cdots \cdot P \mathrm{~d} \eta \mathrm{d} \beta \mathrm{d} \epsilon
$$

These coefficients are not independent of each other. For instance, if we consider the action of a radial (cross-shell) diffusion process, any independent change of, say, I, will be accompanied by dependent changes of mirror point field intensity and energy, determined by the condition of conservation of the first and second invariants $\mu$ and $J$. On the other hand, for a pitch angle scattering mechanism, any independent change $\delta \mathrm{B}$ of $\mathrm{B}$ will be accompanied by a dependent change $\delta \mathrm{I}$ of $\mathrm{I}$, determined by the condition for the particle to remain on the same field line during the scattering process (within one gyroradius, of course) :

$$
\delta \mathbf{I}=\frac{1}{\partial \mathbf{I} / \partial \mathbf{B}} \delta \mathbf{B}
$$

The derivative $\partial \mathrm{V} \partial \mathrm{B}$ is to be taken along the field line at which the scattering occurs. This leads to the relations

$$
\langle\eta\rangle=\frac{\partial I}{\partial B}\langle\beta\rangle,\left\langle\eta^{2}\right\rangle=\left(\frac{\partial I}{\partial B}\right)^{2}\left\langle\beta^{2}\right\rangle \text { e tc. }
$$


for any pitch angle scattering process. This allows a considerable simplification for nearly azimuthally symmetric fields. Let us change variables in the form $\mathrm{L}=\mathrm{L}(\mathrm{I}, \mathrm{B}), \mathrm{B}^{\prime}=\mathrm{B}, \mathrm{X}^{\prime}=\mathrm{X}$. We can rewrite the Fokker Planck equation in these variables, introducing new coefficients, in the form:

$$
\frac{\partial U}{\partial t}+\frac{\partial}{\partial x}(U \dot{\xi})=-\frac{\partial}{\partial L}(U\langle\lambda\rangle)+\frac{1}{2} \frac{\partial^{2}}{\partial L^{2}}\left(U\left\langle\lambda \lambda^{2}\right\rangle\right)-\frac{\partial}{\partial B}(U\langle\beta\rangle)+\cdots \text { etc. }
$$

The relation between $\langle\lambda\rangle$ and $\langle\beta\rangle$ for pitch angle scattering processes will now be of the form:

$$
\langle\lambda\rangle=\frac{\partial L}{\partial B}\langle\beta\rangle,\left\langle\lambda^{2}\right\rangle=\left(\frac{\partial L}{\partial B}\right)^{2}\left\langle\beta^{2}\right\rangle \text {, etc. }
$$

Again, the derivatives are taken along the field line. For a given field line, it is always possible to find a relation $L=L(I, B)$ such that $\partial \mathrm{L} / \partial \mathrm{B}=0$ for all points of this line. This condition, in general, will not be fulfilled for other lines of the same shell. However, for nearly azimuthally symmetric fields, it is possible to find a unique function $\mathrm{L}$ such that $\partial \mathrm{L} / \partial \mathrm{B} \cong 0$ for all field lines of a given shell. This condition implies degeneracy of all shells passing through one field line ${ }^{17}$ (particles mirroring on one field line, populate the same surface during their drift motion, irrespective of their pitch angles or mirror points). For the (internal) geomagnetic field, this function is Mcllwain's L parameter. Using the L-parameter representation instead of $\mathrm{I}$, the corresponding coefficients $\langle\lambda\rangle,\left\langle\lambda^{2}\right\rangle$, etc. vanish in the Fokker-Planck equation, for pitch angle scattering in nearly azimuthally symmetric fields. As there is good evidence that the geomagnetic field does have such properties within about 4-5 earth radii, we shall use this representation in all what follows, and therefore drop the parameter L from our expressions. Before writing our Fokker-Planck 
equation in its final form, we shall re-instate the non-stochastic character to the energy, assuming that the only process for energy change is that of ionization loss, without straggling. This means that the probability $P$ in (12) can be expressed as a delta in energy too:

$$
\mathrm{P}(\mathrm{B}, \mathrm{X}, \mathrm{E}, \beta, \epsilon)=\mathbf{P}^{\prime}(\mathrm{B}, \mathrm{X}, \mathrm{E}, \beta) \delta(\mathrm{E}-\dot{\epsilon} \Delta \mathrm{t})
$$

where $\dot{\epsilon}$ is the energy loss per unit time $(\dot{\epsilon}<0)$.

The final form of our Fokker-Planck equation is therefore, up to the second order term:

$$
\frac{\partial \mathrm{U}}{\partial \mathrm{t}}+\frac{\partial}{\partial \mathrm{X}}(\mathrm{U} \dot{\xi})=-\frac{\partial}{\partial \mathrm{B}}(\mathrm{U}\langle\beta\rangle)+\frac{1}{2} \frac{\partial^{2}}{\partial \mathrm{B}^{2}}\left(\mathrm{U}\left\langle\beta^{2}\right\rangle\right)-\frac{\partial}{\partial \mathrm{E}}(\mathrm{U} \dot{\epsilon})+Q
$$

We can re-write the longitude-convection term as a function of ordinary geographic longitude, $\varphi$, taking into account (9) and (6):

$$
\frac{\partial}{\partial \mathbf{X}}(\mathbf{U} \dot{\xi})=\dot{\varphi}_{0} \tau_{b} \frac{\partial}{\partial \varphi}\left(\frac{\mathbf{U}}{\tau_{b}}\right)
$$

Notice that this is not equal to $\partial / \partial \varphi\left(\dot{\varphi}_{0} \mathrm{U}\right)$, as one would have erroneously obtained, had one written the Fokker-Planck equation in the variable $\varphi$.

\section{DISCUSSION OF SPECIAL CASES}

Let us now discuss equation (14) from the point of view of its physical meaning. First of all, in absence of any diffusion processes and sources, we have:

$$
\frac{\partial \mathbf{U}}{\partial \mathbf{t}}+\frac{\partial}{\partial \mathbf{X}}(\mathbf{U} \dot{\xi})=0=\frac{\partial \mathbf{U}}{\partial \mathbf{t}}+\dot{\varphi}_{0} \tau_{\mathbf{b}} \frac{\partial}{\partial \varphi}\left(\mathrm{U} / \tau_{\mathbf{b}}\right)
$$

which can be written

$$
\frac{\partial}{\partial t}\left(\frac{\mathrm{U}}{\tau_{\mathrm{b}}}\right)+\dot{\varphi}_{0} \frac{\partial}{\partial \varphi}\left(\frac{\mathrm{U}}{\tau_{\mathrm{b}}}\right)=0
$$


Remembering that $\mathrm{U} / \tau_{b}$ is proportional to the directional flux $\mathrm{j}_{0}(3)$, the above expression represents Liouville's Theorem: a flux $j_{0}$, injected at any longitude, proceeds drifting along the shell, maintaining its value unchanged, provided one looks into the correct direction, i.e., along the actual path of the given class of particles. For the earth's field, equation (18) represents the "flight-time spectrometer" effect for a group of particles artificially injected at high altitudes.

If we now integrate equation (14) over one complete longitudinal cycle of $\mathrm{X}$ for a fixed time $t$, the second term on the left vanishes. We obtain:

$$
-\quad \frac{\partial}{\partial t} \oint U d x=-\frac{\partial}{\partial B} \oint U\langle\beta\rangle d X+\frac{1}{2} \frac{\partial^{2}}{\partial B^{2}} \oint U\langle\beta 2\rangle d X-\frac{\partial}{\partial E} \oint U \dot{\epsilon} d X+\bar{Q}
$$

dividing by $\oint \mathrm{dX}$ and calling

$$
\begin{aligned}
U_{A V} & =\frac{\oint U d X}{\oint d X} \\
\dot{\epsilon}_{A V} & =\frac{\oint \dot{\epsilon} U d X}{\oint U d X} \\
\langle\beta\rangle_{A V} & =\frac{\oint\langle\beta\rangle U d X}{\oint U d X} \\
\left\langle\beta^{2}\right\rangle_{A V} & =\frac{\oint\left\langle\beta^{2}\right\rangle U d X}{\oint U d X}
\end{aligned}
$$

We obtain the longitude-independent equation

$$
\frac{\partial U_{A V}}{\partial t}=-\frac{\partial}{\partial E}\left(U_{A V} \dot{\epsilon}_{A V}\right)-\frac{\partial}{\partial B}\left(U_{A V}\langle\beta\rangle_{A V}\right)+\frac{1}{2} \frac{\partial^{2}}{\partial B^{2}}\left(U_{A V}\left\langle\beta^{2}\right\rangle_{A V}\right)+\bar{Q}
$$

This equation is formally equivalent to the time-dependent equation used by several authors ${ }^{10,11,12,14}$. Notice, however, the following remarks: 
(1) The distribution $U_{A V}$ is averaged over the new variabic $X$.

(2) The "coefficients" $\dot{\epsilon}_{\mathrm{AV}},\langle\beta\rangle_{\mathrm{AV}}$ and $\left\langle\beta^{2}\right\rangle{ }_{\mathrm{AV}}$ are not simple cverages over the new coordinate, but they are weighted with the distribution $U$ itself. In other words, they are functionals of the unknown distribution.

All this leads us to the conclusion that a longitude-sverage Fokker-Planck treatment of the problem of trapped particle diffusion is not valid, uniess westrict our description to only those particles which mirror high enough at all longitudes so that their distribution function can be expected a priori as very little longitude dependent. Equation (19) is certainly meaningless as a differential equation for that portion of B -L space, which descends below about $200 \mathrm{~km}$ in the South American Anomaly. This is precisely the domain where the longitude-independent treatment has so far failed to give numerical results compatible with experimental measurements on particle loss rates.

In order to "legalize" the longitude-independent description for such cases in which we know a priori that $U$ will not depend strongly on $X$, we have to evaluate the coefficients of (19) for a distribution $U$ which has the smallest possible longitude dependence. This is given by the solution of $(18): U / \tau_{b} \sim j_{0}=$ const. Taking into account (9), we obtain for the coefficients of the longitudeindependent equation:

$$
\begin{aligned}
\dot{\epsilon}_{\mathrm{A} V} & =\frac{\oint \tau_{\mathrm{b}} \dot{\epsilon} \mathrm{dX}}{\oint_{\tau_{\mathrm{b}} \mathrm{dX}}}=\frac{\oint \dot{\epsilon} \frac{\mathrm{d} \varphi}{\dot{\varphi}_{0}}}{\oint \frac{\mathrm{d} \varphi}{\dot{\varphi}_{0}}} \\
\langle\beta\rangle_{\mathrm{AV}} & =\frac{\oint\langle\beta\rangle \frac{\mathrm{d} \varphi}{\varphi_{0}}}{\oint \frac{\mathrm{d} \varphi}{\dot{\varphi}_{0}}}\left\langle\beta^{2}\right\rangle_{\mathrm{A} V}=\frac{\oint\left\langle\beta^{2}\right\rangle^{\mathrm{d}}}{\varphi_{0}}
\end{aligned}
$$


These expressions are formally equivalent to the longitude-average coefficients used by Hassitt ${ }^{20}$. They represent a longitude average, weighted with the inverse of the local drift velocity. One important difference should, however, be noted: due to the longitude-dependent asymmetry of the real geomagnetic field, integrals along the field line contained in $\dot{\epsilon},\langle\beta\rangle$ and $\left\langle\beta^{2}\right\rangle$ cannot be permuted with the integration over longitude in (20). A correct evaluation of the longitude-independent coefficients therefore requires first, separate evaluation of each coefficient for a certain number of shell field lines, and only then, weighted averaging over longitude.

We must finally insist again that the use of a longitude-independent FokkerPlanck equation for the description of trapped electron diffusion, is not valid at all for those high $\mathrm{B}$ values, for which the atmospheric interactions in the region of the Anomaly will cause a notable longitude dependence of $U$, or $j_{0}$.

We shall now discuss equation (16) for a steady state $\partial U / \partial t=0$, as it is the case for natural inner belt electrons during geomagnetically quiet epochs. We then have

$\frac{\partial}{\partial X}(\mathbf{U} \dot{\xi})=\tau_{b} \dot{\varphi}_{0} \frac{\partial}{\partial \varphi}\left(\frac{U}{\tau_{b}}\right)=-\frac{\partial}{\partial B}(U\langle\beta\rangle)+\frac{1}{2} \frac{\partial^{2}}{\partial B^{2}}\left(\mathrm{U}\left\langle\beta^{2}\right\rangle\right)-\frac{\partial}{\partial E}(\mathrm{U} \dot{\epsilon})+Q$

This will be the basic equation for all what follows: The solution of (21) is subject to a natural boundary condition at the equatorial B-value:

$$
\frac{\partial \mathrm{U}}{\partial \mathrm{B}}=0 \text { for } \mathrm{B}=\mathrm{B}_{0}
$$

At the ends of the field lines in the high atmospheric density region, there is no physical boundary condition at all: as particles enter higher densities, they get 
slowed down by ionization loss and die away in energy space. However, for practical reasons, one can impose an artificial boundary condition

$$
\mathrm{U}=0 \text { for } B \geq B_{c}(X)
$$

which represents a sink of electrons at a level where the field intensity is $B_{c}$ (usually taken as the $B$ value at $100 \mathrm{~km}$ altitude).

The solution of equation (21), with boundary conditions (22) and (23), depends on the source term Q. Unfortunately, very little is known about this term, for inner belt electrons. For sure, its contribution is very small during time intervals of the order of one longitudinal drift period. But this is just the typical time scale of importance when one wants to study the longitude dependence of the electron flux. Of course, one has to start with a given distribution which must be close to an eigenmode of the general equation (16). For the time being we shall neglect the source term in front of all other terms of equation (21). ) .

A useful concept for a qualitative analysis of equation (21) is that of "mirror point flow" along a field line. Let us consider again a population of trapped electrons, mirroring between $\mathrm{B}$ and $\mathrm{B}+\delta \mathrm{B}$, enclosed between two surfaces labeled I and I $+\delta I$ (Fig. 3). According to (1), the number of electrons, drifting through a given longitude (labeled $\mathrm{X}$ ) during a time interval $\delta \mathrm{t}$, will be given by

$$
\mathrm{U} \frac{\mathrm{dX}}{\mathrm{dt}} \delta \mathrm{Y} \delta \mathrm{B} \delta \mathbf{E} \delta \mathrm{t}=\mathrm{U} \dot{\xi} \delta \mathrm{B} \delta \mathbf{E} \delta \mathbf{Y}
$$

The total number of electrons mirroring between $\mathrm{B}$ and the equator, drifting through a given longitude per unit time will then be 


$$
N=\int_{B_{0}}^{B} U \dot{\xi} d B
$$

(We have divided by the constant increments $\delta \mathbf{E} \delta \mathrm{Y}$ ). We shall define as "characteristic mirror point trajectory," the function $B_{m}=B_{m}(X)$, such that the above integral remains constant throughout the drift motion (Fig. 5):

$$
\mathrm{N}=\int_{\mathrm{B}_{0}}^{\mathrm{B}_{\mathrm{m}}(\mathrm{X})} \mathrm{U} \dot{\xi} \mathrm{dB}=\text { coñs } \mathrm{t}
$$

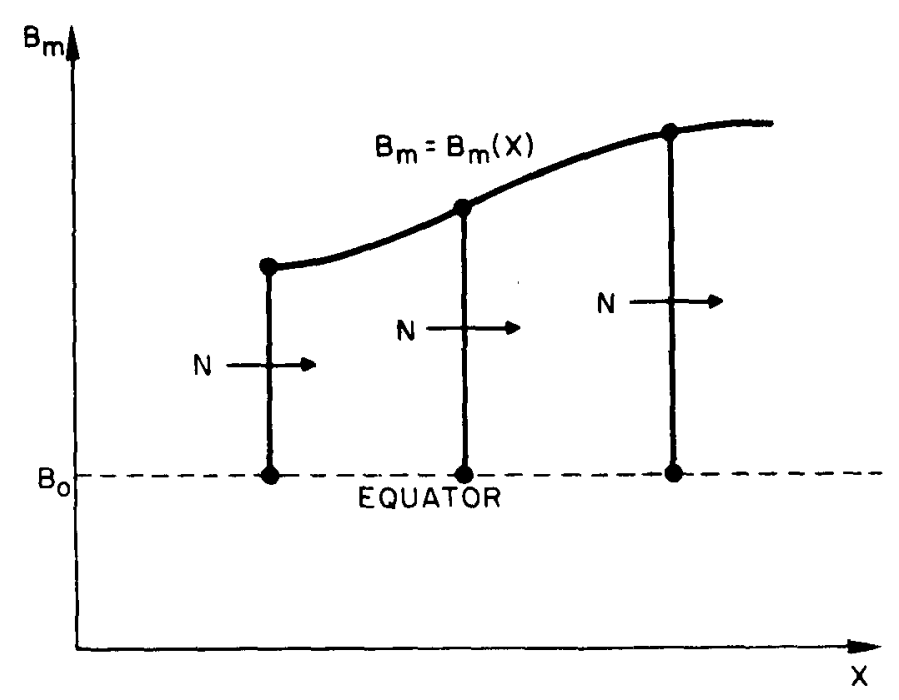

Figure 5

$B_{m}(X)$ of course does not represent the actual path of a particle's mirror point. However, if one neglects energy loss and source contributions, it becomes the average path of the mirror point of a particle in B-X space. This even is true if one does take into account energy loss; only that in this case particle individuality would be lost: a given particle lost out of the energy interval, might be replaced by another one flowing in from a higher energy. Making use of (24), and (10), we now introduce the quantity 


$$
\mathrm{V}=\frac{\mathrm{dB}_{\mathrm{m}}}{\mathrm{d} \varphi}=\frac{\mathrm{dB}{ }_{\mathrm{m}}}{\mathrm{dX}} \frac{\mathrm{dX}}{\mathrm{d} \varphi}=-\frac{\mathrm{p}}{\mathrm{e} \tau_{\mathrm{b}} \dot{\varphi}_{0}} \frac{1}{\mathrm{U} \dot{\xi}} \int_{\mathrm{B}_{0}}^{\mathrm{B}_{\mathrm{m}}(\mathbf{X})} \frac{\partial}{\partial \mathrm{X}}(\mathrm{U} \dot{\xi}) \mathrm{dB}
$$

calling it the "mirror point flow," or rate of change of mirror field per unit longitude. In absence of energy loss and sources, and taking into account (21) and (9), this quantity becomes

$$
\mathrm{V}_{\mathrm{m}}=\bar{\beta}-\frac{1}{2} \partial \mathrm{B}\left(\overline{\beta^{2}}\right)-\frac{1}{2} \bar{\beta} 2 \frac{\partial}{\partial \mathrm{B}}(\ln \mathrm{U})
$$

in which

$$
\bar{\beta}=\frac{1}{\dot{\varphi}_{0}}\langle\beta\rangle \bar{\beta}^{2}=\frac{1}{\dot{\varphi}_{0}}\left\langle\beta^{2}\right\rangle
$$

are mean changes of $\mathrm{B}$ and $\mathrm{B}^{2}$ per unit longitude.

Notice that there are three physically quite distinct contributions to the mirror point "flow." The first term of the right hand of (26) represents a steady increase of $\mathrm{B},(\bar{\beta}$ is positive), i.e., a steady lowering of mirror point aiti-. tude. This contribution comes from the first order, "streaming" term in B (21). In absence of dispersion $\left(\bar{\beta}^{2}=0\right), \bar{\beta}$ is the only contribution to $\mathrm{V}_{\mathrm{m}}$; in that case, $B=B(X)=\int V_{m} d X$ is just the characteristic of the corresponding first order differential equation.

The second and third terms on the right hand side of (26) arise in the dispersion mechanisms. Their presence in (26) clearly shows that dispersion also contributes to a steady, average "flow" of mirror points along field lines. These two terms are governed by the gradients (along field lines) on the coefficient $\bar{\beta}^{2}$, and of $U$, respectively. The gradient of $\beta^{2}$ is always positive, so that the second term in (26) always represents an upward motion of the particle's 
mirror points (back-scattering from the denser atmosphere). If, on the other hand $\partial \mathrm{U} / \partial \mathrm{B}<0$, the third term represents a lowering of the mirror point altitude. If, for instance, $U \bar{\beta}^{2}=$ canst. along a line of force, the contribution from the two dispersion terms is zero: The number of mirror points thrown upwards by the gradient in $\bar{\beta}^{2}$, exactly compensates the number of mirror points streaming downwards due to the gradient of $U$. The only mirror point flow which remains, in this case, is that of the first order term: $V_{m}=\bar{\beta}$.

Notice finally that

$$
\mathrm{C}(\mathrm{B})=\oint \mathrm{U} \dot{\xi} \mathrm{V}_{\mathrm{m}} \mathrm{d} \varphi
$$

represents the total number of electrons of our population crossing a constant-B ring per unit time. The integration is performed around the world along the B-L ring. It is easy to verify that (28) is independent of $B$, for a source-free diffusion. If $\mathrm{V}_{\mathrm{m}}$ is positive at all longitudes (lowering of mirror points), (28) represents the total number of electrons precipitating per unit time through a given constant-B ring.

\section{EXPRESSIONS FOR THE COEFFICIENTS}

In this section we shall obtain expressions for the various coefficients interventing in our fundamental equation (21). First of all, the half-bounce time $\tau_{b}$ is given in terms of the rectified path between mirror points (5):

$$
\tau_{b}(B, \varphi, E)=\frac{1}{v}\left(I+2 B \frac{\partial I}{\partial B}\right)
$$

( $I$ is usually computed in Earth Radii, so (29) must be multiplied by $R_{e}$ ). Expression (29) has absolutely general validity, for any trapping field geometry. 
It offers the big advantage that it only involves computations of $\mathrm{I}$. Results for the real geomagnetic field were cross-checked with numerical integration of the path $S_{b}$ and found to be coincident within high accuracy. For the angular drift velocity $\dot{\varphi}_{0}$, expressions (4) and (6) have to be evaluated numerically.

The energy loss coefficient $\dot{\epsilon}$ is given by $\dot{\epsilon}=1 / \tau_{\mathbf{b}}\{\epsilon\}$ where $\{\epsilon\}$ is the average energy loss per half-bounce:

$$
-\{\epsilon\}=\int_{-S(B)}^{S(B)} \frac{\partial E}{\partial s} \frac{d s}{\sqrt{1-\frac{B^{\prime}(s)}{B}}}
$$

The origin of the field line arc length is taken at the equatorial point. $d s / \sqrt{1-B^{\prime}(s)} \bar{B}$ is the element of trajectory of the electron at a point $s$ where the field is $B^{\prime}(s)$. Using tables given by Berger and Seltzer, ${ }^{21}$ we obtain the following very good approximation:

$$
\left|\frac{\partial E}{\partial s}\right|=10^{-20} \mathrm{~N}_{\text {ef f. ion. }} \mathrm{h}(\mathrm{E})(\mathrm{kev} / \mathrm{cm})
$$

where

$$
h(E)= \begin{cases}2.61+349.0 \mathrm{E}^{-0.844} & \text { for } E<470 \mathrm{kev} \\ 4.54 & \text { for } E \geq 470 \mathrm{kev}\end{cases}
$$

$$
\}
$$

and

$$
\begin{aligned}
& \mathrm{N}_{\text {eff.ion. }}=4.17 \mathrm{~N}_{\text {Argon }}+\mathrm{N}_{\text {oxygen }}+0.881 \mathrm{~N}_{\text {nitrogen }}+ \\
& +0.267 \mathrm{~N}_{\text {helium }}+0.142 \mathrm{~N}_{\text {hydrogen }}
\end{aligned}
$$

is the "effective" atmospheric number density for ionization loss of electrons. 
As we are interested in low $\mathrm{L}$ shells only, we did not include the contribution from atmospheric ions and electrons.

In order to evaluate $\{\beta\}$, the average change of mirror point field $\mathrm{B}$ per half-bounce, we first have to perform the average of collisions over the isotropic azimuthal distribution of scattering angles. Following Welch, Kaufmann and Hess ${ }^{11}$ and slightly changing their notation, we obtain

$$
\widetilde{\beta}=\mathrm{B}\left(\frac{\mathrm{B}}{\mathrm{B}^{\prime}}-\frac{1}{2}\right) \sin ^{2} \theta
$$

$\widetilde{\beta}$ is the change in mirror point field intensity $B$, when the electron's pitch angle scatters an amount $\theta$ at a field position $B^{\prime}$, averaged over all possible azimuthal angles of scattering. Now we have to find the average of $\sin ^{2} \theta$ at the given field point $B^{\prime}$. This will be given by

$$
\overline{\sin ^{2} \theta}=\sum_{i} N_{i} \eta_{i}
$$

where

$$
\eta_{i}=\int_{0}^{\pi / 2} \sin ^{2} \theta \frac{\mathrm{d} \sigma_{i}}{\mathrm{~d} \theta} \mathrm{d} \theta
$$

is the average contribution from one atom of class i. $N_{i}$ are the number densities of the different atmospheric constituents at the field point $B^{\prime} ; \mathrm{d} \sigma \sigma_{i} / d \theta$ are the differential cross sections for screened Coulomb scattering, for each constituent. Using Molière's expression for $\mathrm{d} \sigma_{\mathrm{i}} / \mathrm{d} \theta$ and following closely Welch, Kaufmann, and Hess, ${ }^{11}$ we obtain

$$
\sum_{i} N_{i} \eta_{i}=4 \times 10^{-22} N_{\text {eff. scatt. }} \frac{1+0.167 \ln \sqrt{\frac{E}{m^{2}}\left(\frac{E}{m c^{2}}+2\right)}}{\frac{E}{m c^{2}}\left(\frac{E}{m c^{2}}+2\right)}\left(\mathrm{cm}^{-1}\right)
$$


where

$$
\begin{aligned}
& \mathrm{N}_{\text {eff. scatt. }}=5.18 \mathrm{~N}_{\text {argon }}+\mathrm{N}_{\text {oxygen }}+0.774 \mathrm{~N}_{\text {nitrogen }}+ \\
& +0.058 \mathrm{~N}_{\text {helium }}+0.030 \mathrm{~N}_{\text {hydrogen }}
\end{aligned}
$$

is the "effective" atmospheric number density for screened Coulomb scattering of electrons. Again, the contribution of atmospheric ions and electrons is excluded.

We finally have to perform the integration along the path of a particle from one mirror point to its conjugate:

$$
\{\beta\}=B \int_{-s(B)}^{s(B)}\left(\frac{B}{B^{\prime}(s)}-\frac{1}{2}\right)\left(\sum_{i} N_{i} \eta_{i}\right) \frac{d s}{\sqrt{1-\frac{B^{\prime}(s)}{B}}}
$$

Again following Welch, Kaufman, and Hess, ${ }^{11}$ we obtain $\widetilde{\beta}^{2}$ :

$$
\widetilde{\beta}^{2}=2 B^{2}\left(\frac{B}{B^{\prime}}-1\right) \sin ^{2} \theta
$$

Therefore:

$$
\left\{\beta^{2}\right\}=2 B^{2} \int_{-s(B)}^{s(B)}\left(\frac{B}{B^{\prime}(s)}-1\right)\left(\sum_{i} N_{i} \eta_{i}\right) \frac{d s}{\sqrt{1-\frac{B^{\prime}(s)}{B}}}
$$

If we now call:

$$
\begin{aligned}
& S_{1}(B, \varphi)=\int_{-s(B)}^{s(B)} \frac{B}{B^{\prime}(s)} \frac{N_{\text {eff. scatt. }}(s, \varphi)}{\sqrt{1-B^{\prime}(s) / B}} \mathrm{ds}\left(\mathrm{cm}^{-2}\right) \\
& S_{2}(B, \varphi)=\int_{-s(B)}^{s(B)} \frac{N_{\text {eff. scatt. }}(s, \varphi)}{\sqrt{1-B^{\prime}(s) / B}} \mathrm{ds}\left(\mathrm{cm}^{-2}\right)
\end{aligned}
$$




$$
\begin{gathered}
S_{2}^{\prime}(B, \varphi)=\int_{-s(B)}^{s(B)} \frac{N_{\text {eff. ion }}(s, \varphi)}{\sqrt{1-B^{\prime}(s) / B}} d s\left(\mathrm{~cm}^{-2}\right) \\
\left.K(B, E)=4 \times 10^{-22} B \frac{1+0.167 \ln \sqrt{\frac{E}{m^{2}}\left(\frac{E}{m^{2}}+2\right)}}{\frac{E}{n^{2}}\left(\frac{E}{m^{2}}+2\right)} \text { (gauss } \mathrm{cm}^{2}\right) \\
\text { (C) } C(E)=10^{-20} \mathrm{~h}(E) \quad\left(k e v \mathrm{~cm}^{2}\right)
\end{gathered}
$$

we can summarize expressions $(35),(36)$ and $(30)$ in the form

$$
\begin{aligned}
& \{\beta\}=\mathrm{K}\left(\dot{\mathrm{S}}_{1}-\frac{1}{2} \mathrm{~S}_{2}\right) \\
& \left\{\beta^{2}\right\}=2 \mathrm{BK}\left(\mathrm{S}_{1}-\mathrm{S}_{2}\right) \\
& \{\epsilon\}=\mathrm{CS}_{2}^{\prime}
\end{aligned}
$$

In the region of very high atmospheric densities, as, say, below $200 \mathrm{~km}$, the first two coefficients in (37) are related to each other. Suppose an effective density for scattering of the type

$$
N_{\text {eff. scatt. }}=N_{0}(\varphi) \exp \left(\frac{B-B_{a}(\varphi)}{\Delta B(\varphi)}\right)
$$

where $B_{a}(\varphi)$ is the $B$ value at the $100 \mathrm{Km}$ level, and

$$
\Delta \mathrm{B}(\varphi) \ll \mathrm{B}_{\mathrm{a}}(\varphi)
$$

With this expression, it can be shown that 


$$
S_{1} \simeq S_{2} \simeq N_{0} \exp \frac{B-B_{a}(\varphi)}{\Delta B(\varphi)} \frac{\sqrt{\pi \frac{\Delta B(\varphi)}{B}}}{\frac{\partial B}{\partial s}}
$$

and

$$
\mathrm{S}_{1}-\mathrm{S}_{2} \simeq \mathrm{S}_{1} \frac{\Delta \mathrm{B}(\varphi)}{2 \mathrm{~B}}
$$

$\partial B / \partial s$ is the average gradient of $B$ along the field line, in the region of interest. Taking into account (55), the following relation between the coefficients $\{\beta\}$ and $\left\{\beta^{2}\right\}$ holds:

$$
\left\{\beta^{2}\right\}=2 \triangle B\{\beta\}
$$

This is similar to a relation found by Walt and McDonald ${ }^{12}$ for the coefficients of a time dependent (but longitude-independent) Fokker-Planck equation, set up in terms of mirror point altitudes, in the region of high atmospheric densities.

Notice that with (38), the mirror point flow (26) at low altitudes becomes:

$$
\mathrm{V}_{\mathrm{m}} \simeq-\Delta \mathrm{B} \bar{\beta} \frac{\partial}{\partial \mathrm{B}}(\ln \mathrm{U})
$$

Physically, the cancellation of the first term with the second term in (26) means that the upstream of mirror points due to diffusion from the denser atmosphere ("backscattering" at lower altitudes) exactly compensates the downstream due to the first order term. What is left, is a mirror point streaming, entirely due to diffusion, and which may be upwards or downwards, according to the gradient of the actual electron distribution, along a line of force. A similar result was obtained numerically by MacDonald and Walt ${ }^{13}$.

Notice at once that for a distribution function inversely proportional to the effective atmospheric density 


$$
U \sim\left(N_{\text {eff }} . \operatorname{scatt}\right)^{-1} \sim \exp \left(\frac{B-B_{a}(\varphi)}{\Delta \bar{B}(\varphi)}\right)
$$

which is close to a steady state solution of equation (21), expression (39) becomes

$$
\mathrm{V}_{\mathrm{m}} \simeq \bar{\beta}
$$

at low altitudes. This is enough to predict qualitatively the behavior of the average "path" of the mirror point of an electron which drifts through the South American Anomaly: while the particle is still well West of the Anomaly, the mirror point will follow a constant-B ring. As the particle approaches the Anomaly, this ring will dip into higher atmospheric densities, and the mirror point will start "flowing" downwards (towards higher $B$ values, because $V_{m}$ in (40) is always positive). If the particle was initially high enough in altitude (at low B), it will emerge again from the Anomaly, though with a higher mirror point B-value, eventually populating the B-L region which was voided in the Anomaly. After a sufficient number of drifts around the earth, the mirror point trajectory may start low enough on the West side, in order to succeed precipitating below the $100 \mathrm{~km}$ level in the Anomaly. Of course, during this whole process, the energy of a given electron decreases steadily. Electrons should mainly precipitate on the West side of the Anomaly. This purely qualitative analysis also shows that one should expect a diurnal variation of the electron distribution in the Anomaly, related to the diurnal variation of the atmosphere in that region.

Notice finally, that according to this picture, replenishment should occur right at, or shortly "after," i.e., East of the Anomaly. Diffusion into the 
"shadow" region far East of the Anomaly is negligible, for pure Coulomb scattering. We may conclude our discussion by pointing out that in the picture described above, there is a steady flow of particles out of the lower B region of a shell, towards higher $B$ values, and from there, into the atmosphere at the Anomaly. In an equilibrium state, this loss must be exactly balanced by a continuous injection from a source mainly effective in the lower B region. In absence of such a source, i.e., in a non-equilibrium state, this flow and subsequent loss should determine the lifetime of the trapped radiation in question.

\section{ACKNOWLEDGMENTS}

We are indebted to Drs. W. N. Hess, D. Stern, J. Herring and M. Liwshitz for valuable discussions and critical remarks.

\section{REFERENCES}

1. Yoshida S., G. H. Ludwig and J. A. Van Allen, "Distribution of Trapped Radiation in the Geomagnetic Field," Journal of Geophys. Res. 65, 807-813 (1960)

2. Vernov, S. N., E. V. Gorchakov, Yu. I. Logachev, V. E. Nesterov, N. F. Pisarenko, I. A. Savenko, A. E. Chudakov and P. I. Shavrin, "Investigations of Radiation During Flights of Satellites, Space Vehicles and Rockets, Suppl. J. Phys. Soc. of Japan 17, 162 (1962)

3. Imhof, W. L., and R. V. Smith, "Longitudinal Variations of High Energy Electrons at Low Altitudes," J. Geophys. Res. 70, 569-577 (1965)

4. Paulikas, G. A. and S. C. Freden, "Precipitation of Energetic Electrons into the Atmosphere," Journal of Geophys. Res. $\underline{69}$, 1239-1249 (1964)

5. Mihalov, J. D., F. S. Mozer and R. S. White, "Artifically Injected Electrons at Low Altitude" Journal of Geophys. Res. $\underline{69,}$ 4003-4013 (1964) 
6. Freden, S. C. and G. A. Paulikas, "Trapped Protons at low Altitudes in the South Atlantic Magnetic Anomaly," Journal of Geophys. Res. $\underline{69}$, 1259-1269 (1964)

7. Heckman, H. H. and G. H. Nakano, "Direct Observations of Mirroring Protons in the South Atlantic Anomaly," Space Research V, 329-342 (1965)

8. Ghielmetti, H. S., N. Becerra, A. M. Godel, H. Heredia and J. G. Roederer, "Enhancement of the X-Ray Intensity at Balloon Altitudes in the South American Anomaly," Phys. Rev. Letters 12, 388-390 (1964)

9. Williams, D. J. and J. W. Kohl, "Loss and Replenishment of Electrons at Middle Latitudes and High B-Values," Journal of Geophys. Res. $\underline{70}$, 4135$4150(1965)$

10. MacDonald, W. M. and M. Walt, "Distribution Function of Magnetically Confined Electrons in a Scattering Atmosphere," Annals of Physics $\underline{15}$, $44-62(1961)$

11. Welch, J. A., R. L. Kaufmann and W. N. Hess, "Trapped Electron Time Histories for $\mathrm{L}=1.18$ to $\mathrm{L}=1.30, "$ Journal of Geophys. Res. $\underline{68}, 685-699$ (1963)

12. Walt, M. and W. M. MacDonald, "Diffusion of Electrons in the Van Allen Radiation Belt, I: Treatment of Particles with Mirroring Points at High Altitude," Journal of Geophys. Res. 67, 5013-5024 (1962)

13. MacDonald, W. M. and M. Walt, "Diffusion of Electrons in the Van Allen Radiation Belt, II: Particles with Mirroring Points at Low Altitude," Journal of Geophys. Res. 67, 5025-5034 (1962)

14. Anderson, A. D., G. E. Crane, W. E. Francis, L. L. Newkirk and M. Walt, "Theoretical Investigation of Geomagnetically Trapped Electrons from High Altitude Nuclear Explosions," Lockheed Missiles and Space Company Report LMSC 895355 (1964) 
15. McIlwain, C. E., "Coordinate for Mapping the Distributions of Magnetically Trapped Particles," Journal of Geophys. Res. 666, 3681-3691 (1961)

16. Northrop, T. G. and E. Teller, "Stability of the Adiabatic Motion of Charged Particles in the Earth's Field," Phys. Rev. 117, 215-225 (1960)

17. Stone, E. C., "The Physical Significance and Application of $L, B_{o}$ and $R_{O}$ to Geomagnetically Trapped Particles," Journal of Geophys. Res. $\underline{68}$, $4157-4166(1963)$

18. Roederer, J. G., "On the Adiabatic Motion of Energetic Particles in a Model Magnetosphere,"

19. Northrop, T. G., "The Adiabatic Motion of Charged Particles," Interscience Publishers (1963)

20. Hassitt, A., "The Drift Velocity of Trapped Particles," Journal of Geophys. Res. $\underline{70}, 535-540$ (1965)

21. Berger, M. J. and S. M. Seltzer, NASA Report SP-3012 (1964) 
LONGITUDE DEPENDENCE OF GEOMAGNETICALLY

TRAPPED ELECTRONS

PART II

by

Juan G. Roederer*

Goddard Space Flight Center

Greenbelt, Maryland

Jasper A. Welch**

Air Force Systems Command

Los Angeles, California

and

James V. Herod***

Air Force Weapons Laboratory

Kirtland AFB, New Mexico

\footnotetext{
*National Academy of Sciences - National Research Council, Senior Post Doctoral Research Associate. Present address: Centro Nacional de Radiación Cósmica, Peru 272, Buenos Aires, Argentina

${ }^{\star \star}$ Lt. Colonel, United States Air Force

$\star \star \star$ Captain, United States Army
} 


\begin{abstract}
The Fokker-Planck equation, discussed in Part I of this paper, was integrated numerically for a stationary case, for low L shells. A computer code was developed, which gives detailed information on particle shell geometry, and on longitude dependence of drift velocities and bounce periods. The coefficients representing longitudinal drift, ionization loss and multiple Coulomb scattering in the Fokker-Planck equation were computed numerically.

The "eigenmode" electron distribution function for the longitude dependent problem was determined, and electron fluxes, energy spectra, B-dependence and mirror point trajectories were calculated for the area of the anomaly. The results are in general agreement with satellite observations obtained in the vicinity of the anomaly. They do not, however, explain the observed replenishment which occurs at all other longitudes east of the anomaly. This latter effect must be caused by a pitch angle scattering mechanism other than multiple Coulomb interactions with atmospheric atoms.
\end{abstract}




\section{LONGITUDE DEPENDENCE OF GEOMAGNETICALLY}

\section{TRAPPED ELECTRONS}

\section{PART II}

\section{INTRODUCTION}

Pitch angle scattering is the main physical mechanism responsible for the shape of the equilibrium distribution of electrons in the inner radiation belt. These particles, if continuously injected into a given L-shell by a source, or transferred to it by radial diffusion, will gradually change their mirror points by such a pitch angle scattering mechanism, until a stationary state is achieved, in which the mirror point distribution represents an eigenstate of the corresponding mathematical diffusion problem. In this stationary state, injection rate equals the rate of removal, the latter being determined by the rate of transference of electrons to neighboring shells, and by the flow of mirror points into the dense part of the atmosphere. It is believed that for low L shells, radial transfer is much less efficient than atmospheric loss.

In the inner magnetosphere there are at least two types of pitch angle scattering processes: multiple Coulomb scattering with atmospheric constituents and resonant interactions with VLF electromagnetic or ion waves 1, 2, 3,4. The first one must obviously be dominant at very low $L$ values; the second one becomes more and more important as one goes to higher $\mathrm{L}$ shells. There are, however, reasons to expect that "electromagnetic" scattering is still effective at low L-shells, coexisting with Coulomb scattering. If this is the case, it should be difficult to determine experimentally how much of the scattering is of 
non-Coulombic origin. The study of the longitude dependence of electron fluxes offers a possibility: Coulomb interactions are confined to a rather narrow band of longitudes in the South American Anomaly, and so must be the corresponding effect on the electron distribution. Electromagnetic interactions, instead, are not expected to have such a strong longitude dependence; the associated pitch angle diffusion should occur more or less uniformly over all longitudes. One possible way to obtain information about this electromagnetic scattering mechanism at low L-shells is, therefore, to predict theoretically the Coulomb contribution to the longitude effect of the electron flux, and then to compare it with experimental results, blaming all observed differences on the electromagnetic scattering process.

In Part I of this paper, the theoretical background for the study of the longitude dependence of a stationary electron distribution at low L-shells was given, under the assumption that multiple Coulomb scattering is the only interaction process present. In Part II we shall present results of numerical calculations for some particular $\mathrm{L}$ values, and compare them with the scarce experimental material so far available on this subject.

\section{FIELD GEOMETRY}

In order to solve the theoretical problem of longitude dependence of trapped electron distributions, it is first necessary to evaluate the coefficients intervening in the diffusion equation (21) of Part I. These coefficients, given in equations (37), contain integrals $\left(S_{1}, S_{2}\right.$ and $\left.S_{2}^{\prime}\right)$ of functions which vary many orders of magnitude over the domain of integration along the field line. Therefore, the first requirement for these calculations is an accurate description of 
the geometry of magnetic shells, as well as the determination of the longitude dependence of all intervening kinetic variables, such as drift velocities and bounce periods.

For particles mirroring at low altitudes in the South American Anomaly, changes of a few kilometers in altitude of magnetic shells may induce quite appreciable changes in the numerical results of theoretical diffusion calculations. Considerable accuracy is therefore required. Of course, there will always be a natural limitation imposed by the degree of accuracy in the geomagnetic field description. However, there are other more or less controllable sources of errors, both systematic and random, which are inherent to the numerical methods used in shell geometry and particle kinematics computations, and which must be reduced to a minimum.

One of such sources is related to the question of shell splitting ${ }^{5}$. This shell splitting amounts to only a few kilometers at the equator, for $L \simeq 1.2$. Nevertheless, such difference of a few kilometers may be of importance to mechanisms of atmospheric interactions, where it can compete with the scale height of atmospheric density. Special care must then be taken, when a particle shell is to be defined. For instance, if one is interested in the evolution of a group of particles mirroring far away from the equator, i.e. at high B-values, the field lines on which these particles will mirror must be defined by the proper $B_{m}, L$ (or $\mathrm{B}_{\mathrm{m}}, \mathrm{I}$ ) ring, and not by the equatorial ring of the corresponding L-shell. The differences which result from picking shells by their equatorial points instead of some higher B-value points, may be as much as $10-20 \mathrm{Km}$ in the region of the Anomaly. The corresponding differences in longitude dependence of drift 
velocities and bounce periods are, percentage wise, even higher $(6-10 \%$ for $\mathrm{L}=1.25$ ); finally, the equatorial $\mathrm{B}$-value of a shell picked at high $\mathrm{B}$ becomes appreciably longitude dependent (up to $6-7 \%$ for $\mathrm{L}=1.25$ ), and so does the equatorial pitch angle of a given particle mirroring at higher latitudes.

Most of the longitudinal variations occur in the South American Anomaly, where, on the other hand, most of the atmospheric interactions take place. Unfortunately, this is the region where higher multipoles of the field expansion are felt most intensely, being therefore more exposed to errors of the particular field description used.

A computer program was set up which meets all requirements of accuracy and speed ${ }^{6}$, giving detailed information on field line geometry for a given L-shell. In addition, it calculates the geometric factors intervening in drift velocities and bounce paths, using expressions (4) and (5) of Part I, respectively. As an example, the longitude dependence of the angular drift velocity (in arbitrary units) and that of the bounce path, for the $L=1.25$ shell and two mirror point $B$-values, are shown in Figs. 1 and 2, respectively. When these curves are to be compared with previously calculated ones ${ }^{7,8}$, one has to bear in mind that longitude dependence of drift velocities and bounce paths very critically depend on how the particle shell was originally defined. The correct calculation requires picking the L-shell at precisely the $B_{m}$ points for which these quantities are to be determined. This is accomplished in the present code.

\section{CALCULATION OF THE COEFFICIENTS}

Once the field lines are defined on a given L-shell, the integrals for the coefficients (37) have to be computed on each field line, and for different mirror 


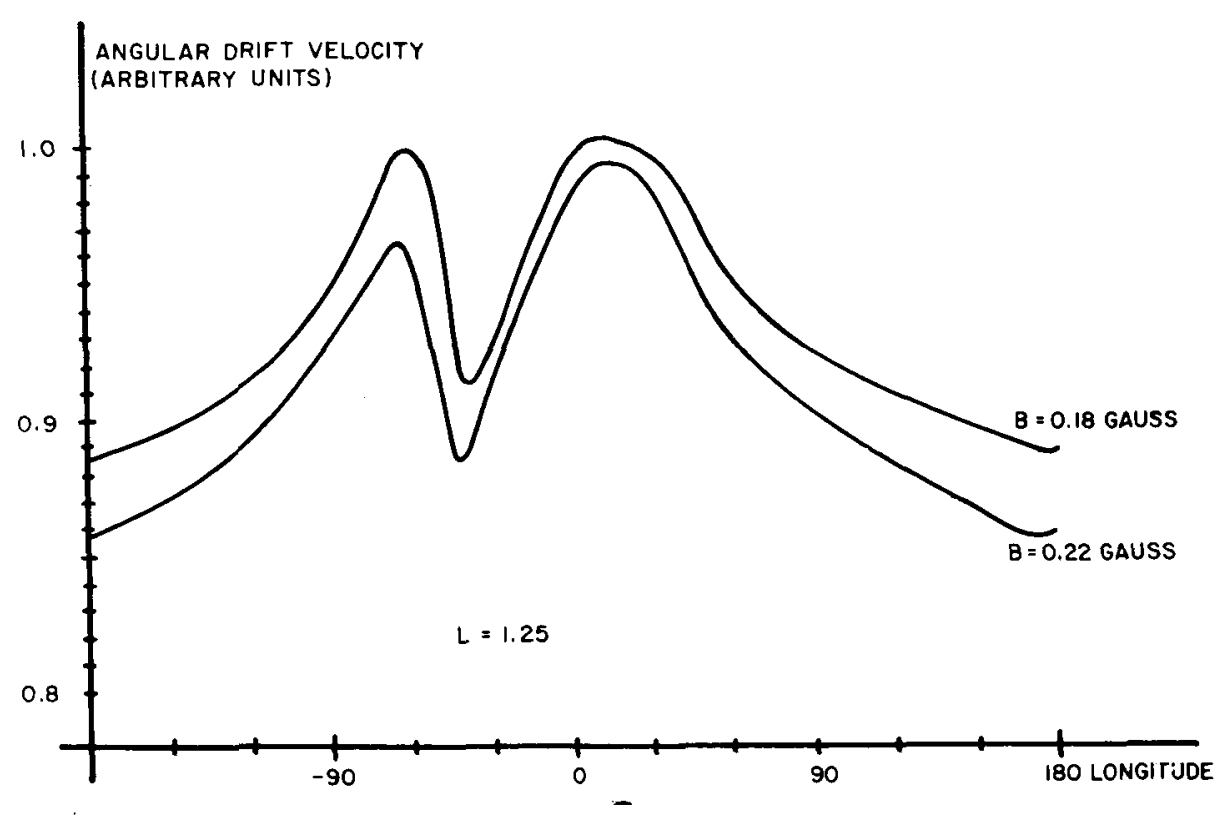

Fig. 1. Angular drift velocity as a function of the longitude of the equatorial point, for particles mirroring at $B=0.18$ and 0.22 gauss, for $L=1.25$.

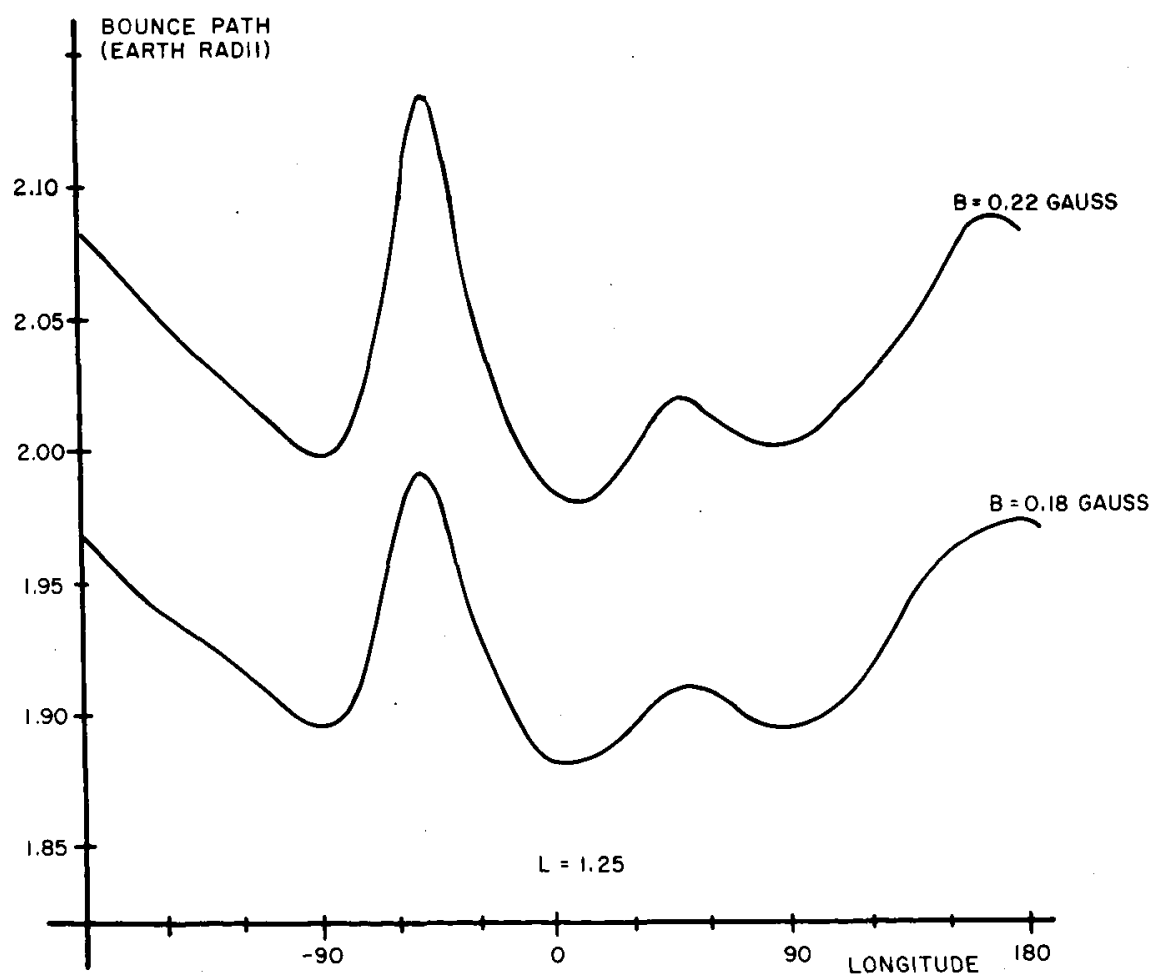

Fig. 2. Bounce path between conjugate mirror points, of electrons mirroring at $B=0.18$ and 0.22 gauss, for $L=1.25$. To obtain the half-bounce period, divide by the particle velocity in earth radii/sec. 
point field B-values. These B-mesh points were defined at geodetic altitudes spaced roughly proportional to $1 / 2-1 / 5$ of the local atmospheric scale height, at the low altitude end of each field line. The mesh was then conveniently completed up to the equatorial point. On the other side of the equatorial point, the mesh was defined as the magnetically conjugate image of the original one. The B-mesh thus obtained had variable, longitude dependent cell size. In total, 100 pairs of B-mesh points were defined on each field line. These field lines were spaced at longitude intervals of 5 or 3 degrees, decreasing to steps of $1^{\circ}$ in the region of the anomaly.

Atmospheric number densities intervening in (32) and (34) depend on local time at altitudes above $150 \mathrm{Km}$. In order to take this into account, and to find its effect on the electron distribution, all computations were performed for two different local times in the region of the anomaly $(0400$ and $1400 \mathrm{LT})$. Each field line was assigned a mean local time according to its longitudinal position on the shell. The Harris-Priester atmosphere ${ }^{9}$ was used; and two extreme cases of low and high solar activity were considered. For a given L-shell, the energyindependent parts of the coefficients were written on magnetic tape, for each of the four combinations night/day in the anomaly and low/high solar activity. A description of the computer code will be published elsewhere.

\section{NUMERICAL SOLUTION OF THE DIFFUSION EQUATION}

As a first step, mainly to check the coefficients calculated in the previous section, the time dependent equation (19) was solved using coefficients averaged over local time and longitude as prescribed in expression (20). Results are in general agreement with previous calculations ${ }^{10}$, in particular, for the decay of 
the Starfish injection ${ }^{11}$. The disagreement with experimental results for higher B-values, found by Welch et $\mathrm{al}^{10}$ and by Walt and Newkirk ${ }^{11}$, still subsists in our calculations (theoretical calculations always give much faster decays at high B-values, than experimentally observed).

We now turn to the numerical solution of equation (21), for a stationary state. In order to integrate equation (21), the source term $Q$ must be known. This, unfortunately, is not possible for the time being. However, injection during intervals of time comparable to a typical drift period must be expected to be rather small at low L-shells. We shall therefore neglect the contribution from $Q$, always keeping in mind that with this assumption, a true steady state can never be achieved in the numerical calculations. The error committed should be small in comparison with other inaccuracies (atmospheric density, magnetic field, etc.).

To solve numerically eq. (21) under these conditions, we have to start with a given initial distribution $U$ at an initial longitude $\varphi_{i}$. Proceeding once around the world with the numerical integration, until the starting longitude is again reached, one ends up with a distribution which, in general, will be different from the initial one. In particular, in absence of a source term $Q$, the integral flux of particles will have diminished due to the action of the sink at low altitudes. We shall arbitrarily call an "eigenmode" of eq. (21), a distribution for which this "one-time-around" difference is minimum (for a true eigenmode, this difference is zero).

The point is, then, to start at $\varphi_{i}$ with a distribution which already is as close as possible to an eigenmode and to proceed integrating around the earth a sufficient number of times until the "one-time-around" difference have become small enough. 
For practical purposes it is not necessary to integrate every time around the whole world. It will be sufficient to consider a longitude interval, centered at the anomaly, with a width determined by the range of B-values of interest. For instance, for the $\mathrm{L}=1.25$ shell, it is sufficient to consider the longitude interval between approximately $-70^{\circ}$ and $-18^{\circ}$, if one wants to explore the region $B \leq 0.270$ gauss. Outside this longitude interval, the effect of multiple Coulomb scattering is negligible, due to the high altitude of the $\mathrm{B}-\mathrm{L}$ rings under consideration.

The procedure to obtain the stationary, longitude dependent electron distribution is sketched in Fig. 3 , for the case $\mathrm{L}=1.25$. We have used the solution of equation (19) obtained for long periods of time after injection, as the initial distribution for the longitude dependent equation (21), injected right at the center of the anomaly $\left(\varphi_{i}\right)$. The output distribution obtained at $\varphi=-20^{\circ}$ is reinjected at $-70^{\circ}$, and this process is then repeated several times, until the percentage change of $U$ per integration cycle $\left(\Delta \mathrm{U}=\mathrm{U}_{-20}-\mathrm{U}_{-70}\right)$ reaches a constant value. The resulting distribution $U$ is then adopted as "eigenmode" of the longitude dependent problem. In the real case, the small differences $\Delta U(<0)$ must be cancelled by the longitude integral of the injection rate $\int Q d \varphi$. Numerical results have shown that, in general, the distribution $U$ which builds up in the region of replenishment east of the Anomaly, is only very weakly dependent on the initial distribution injected at $\varphi_{i}$. In other words, the eigenmode is achieved very fast in this high-B region.

The computer program for the integration of eq. (21) was designed in such a way as to perform the reinjections as many times as wanted. In this program, 


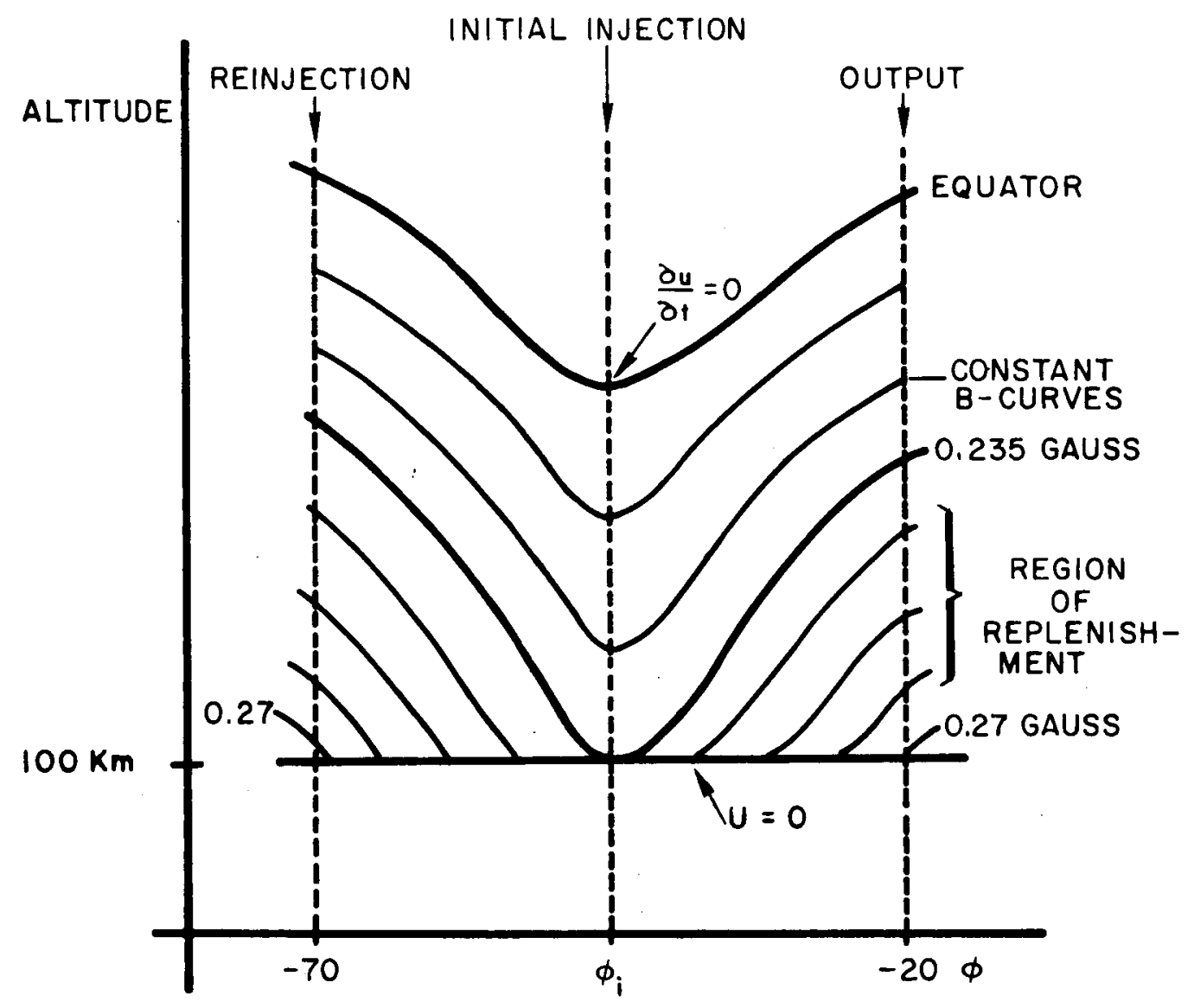

Fig. 3. Sketch to illustrate the procedure of numerical integration of equation (21) (Part I). 
the initial distribution is read in, and the energy dependent parts of the coefficients are computed. The field-geometric part of these coefficients is then read in from magnetic tape, for the first pair of field lines, and the associated difference equations are solved. This procedure is continued until a prefixed longitude east of the anomaly is reached, at which integration stops. The result is reinjected at another fixed longitude west of the anomaly, and the whole process is repeated.

In order to better interpret the physical implications of the procedure to integrate eq. (21), outlined above, we go back to the general equation (16). If we suppose a sudden injection at a time $t_{i}$ and a longitude $\varphi_{i}$, of the form

$$
\mathbf{Q}=\mathbf{U}_{\mathbf{i}} \delta\left(\mathbf{t}-\mathbf{t}_{\mathbf{i}}\right) \delta\left(\varphi-\varphi_{\mathbf{i}}\right)
$$

and integrate equation (16) from $t_{i}-\delta t$ to infinity, we have, taking into account (17):

$$
\tau_{\mathrm{b}} \dot{\varphi}_{0} \frac{\partial}{\partial \varphi}\left(\frac{W}{\tau_{\mathrm{b}}}\right)=-\frac{\partial}{\partial \mathrm{B}}(\mathrm{W}\langle\beta\rangle)+\frac{1}{2} \frac{\partial^{2}}{\partial \mathrm{B}^{2}}\left(\mathrm{~W}\left\langle\beta^{2}\right\rangle\right)-\frac{\partial}{\partial \mathrm{E}}(\mathrm{W} \dot{\epsilon})
$$

where

$$
W(B, \varphi, E)=\int_{t_{i}}^{\infty} U(B, \varphi, E, t) d t
$$

According to (3) and (42), the quantity

$$
j_{W}=\frac{B^{2}}{2 \pi \tau_{b}} \mathbf{W}(B, \varphi, E)
$$

represents the total number of electrons of energy between $E$ and $E+d E$ which have passed once in their longitudinal drift through the longitude $\varphi$, per unit area perpendicular to their pitch angle. This clearly defines the physical meaning of $W$. 
Notice now that eq. (41) is formally identical to the longitude dependent equation (21), in absence of a source term Q. In conclusion, the solution of eq. (21) for $Q=0$, and for a given initial distribution $U$ injected at $\varphi_{i}$, physically represents, at each longitude, the total number of electrons which would have passed through that longitude on their first trip around the earth after injection (it does not contain any information about electrons going through on their second, third, etc., time around, nor does it say what the distribution was at any given time $\mathrm{t}$ ).

\section{RESULTS}

Before analyzing general results, we turn to the discussion of the mirror point flow, given in section IV of Part I. Once the solution of eq. (21) has been obtained, one can integrate (25) in order to find the "mirror point trajectories" $\mathrm{B}_{\mathrm{m}}=\mathrm{B}_{\mathrm{m}}(\varphi)$. Fig. 4 shows the result for $600 \mathrm{kev}$ electrons, for $\mathrm{L}=1.25(0400 \mathrm{LT}$ in the anomaly, low solar activity). All qualitative predictions, given in the last section of Part I are clearly confirmed. Notice the "window" in B which feeds the B-region originally wiped out of particles in the anomaly, and watch how higher mirror points are gradually lowered in altitude, as they drift through the anomaly. Constant altitude curves are reproduced in order to show at which altitudes most of the mirror point flow occurs. It can be seen that the slope $\left(V_{m}\right)$ of the mirror point trajectories is nearly constant along constant altitude curves.

Figs. 5 and 6 show general results of the integration of eq. (21). The computed, directional flux $j_{0}$ of $300 \mathrm{kev}$ electrons is plotted for different L-values as a function of longitude, normalized for different B-values outside the anomaly. Notice the following features: (a) Replenishment via multiple Coulomb scattering 


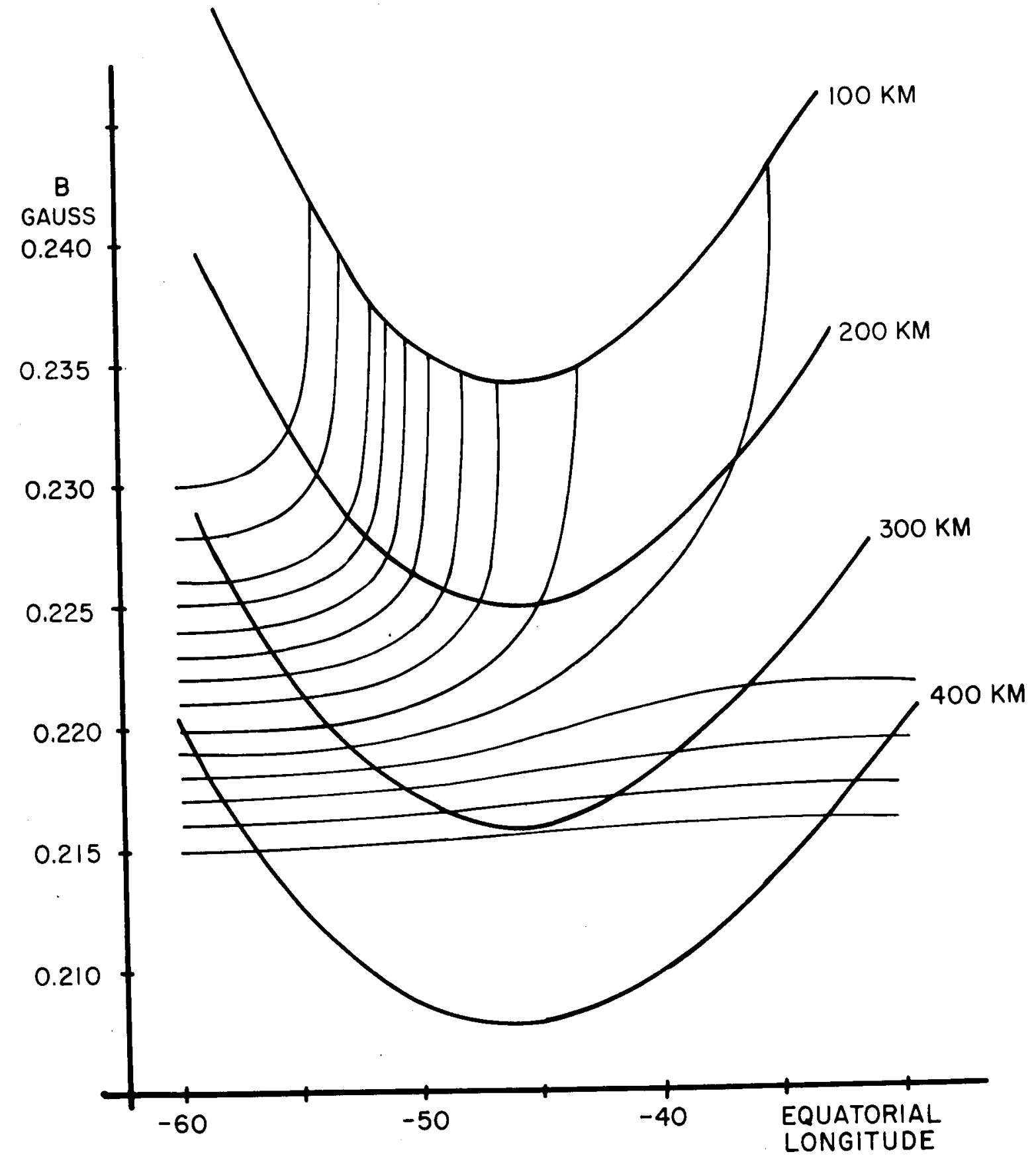

Fig. 4. Mirror point trajectories of $600 \mathrm{kev}$ electrons (average energy of electrons in the region of replenishment). Constant altitude curves are shown. Notice the narrow "window" of mirror point field intensities around $B=0.218$ gauss, west of the an omaly, which "feeds" the region of replenishment on the east side. 


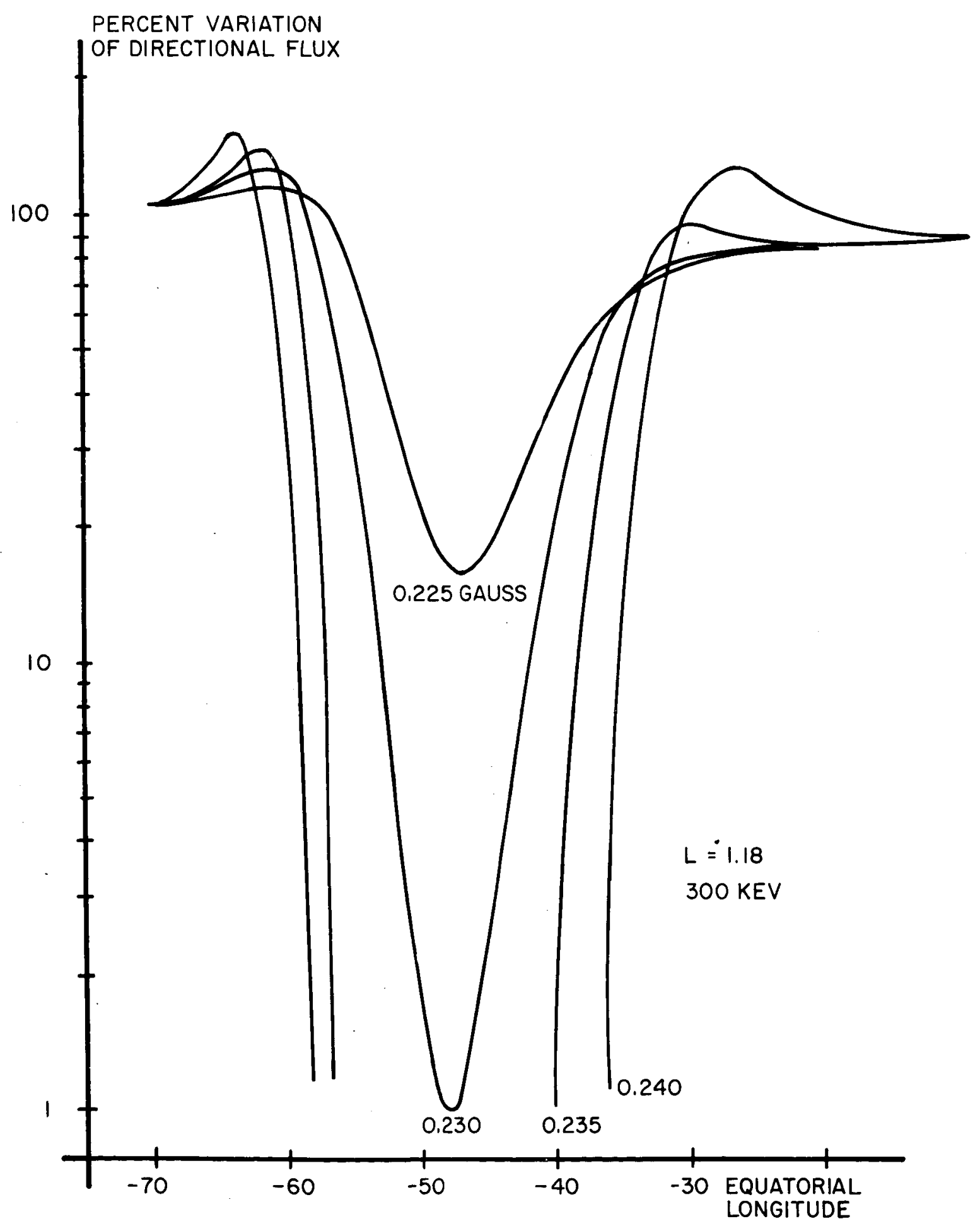

Fig. 5. Longitude dependence of the directional flux $i$ of $300 \mathrm{kev}$ electrons mirroring at different $B$-values, for $L=1.18$. 


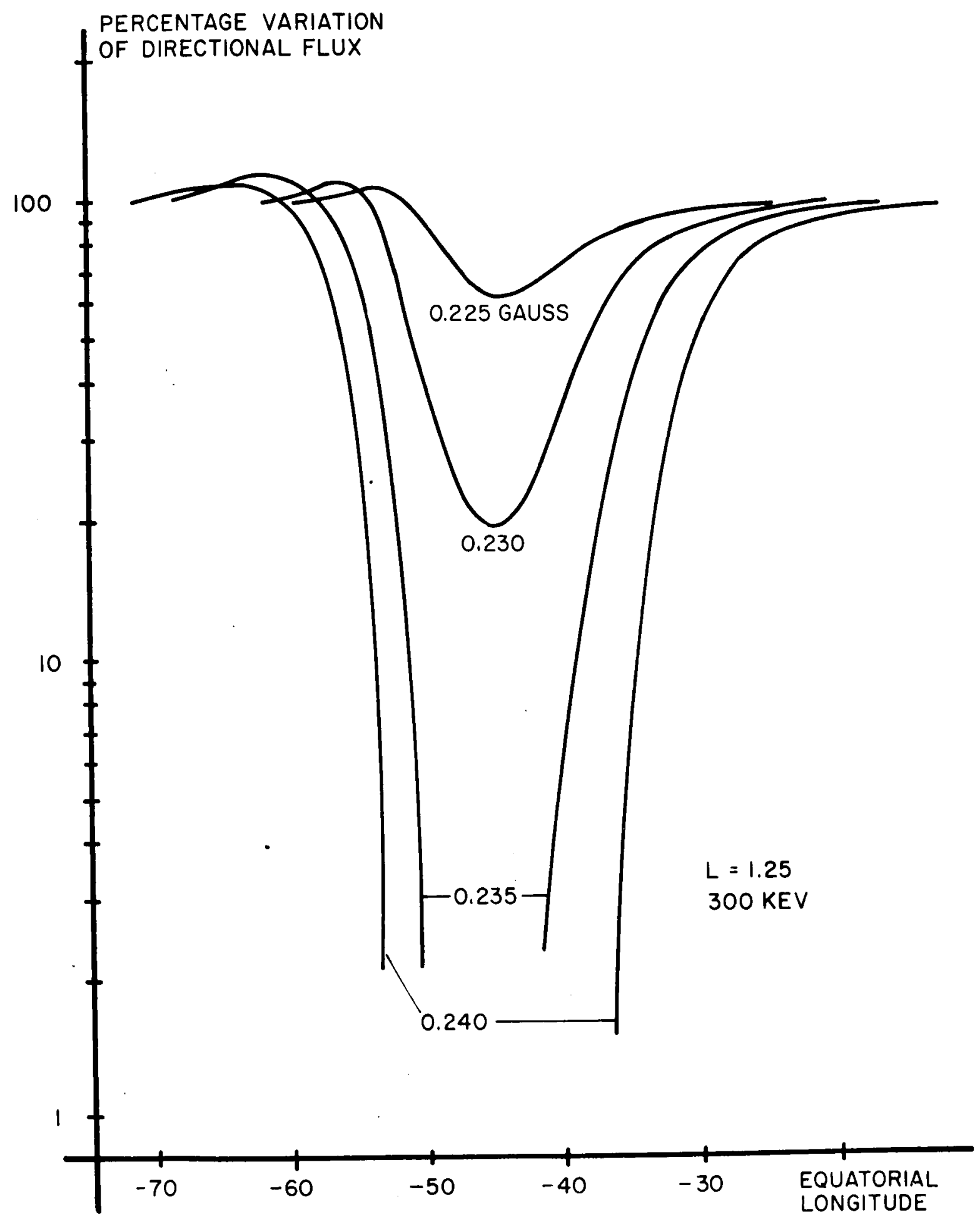

Fig. 6. Longitude dependence of the directional flux $;$ of $300 \mathrm{kev}$ electrons mirsoring at different $B$-values, for $L=1.25$. 
occurs within $20-25^{\circ}$ east of the point of emergence from the $100 \mathrm{Km}$ level of the corresponding B-L ring. (b) The process is not symmetric with respect to the anomaly: scattering out (loss) of a B-interval west of the anomaly is faster, than scattering in (replenishment) on the east side. This asymmetry is more pronounced than the natural asymmetry of B-L rings. (c) At lower L values, for which B-L rings submerge into, and emerge from, the dense atmosphere in much steeper fashion, a "piling up" of particles occurs just prior to the decrease, and just after replenishment. This piling up shows up, but less pronounced, in the omnidirectional fluxes.

Next, we reproduce in Figs. 7 and 8 the energy spectra of electrons mirroring at different, high B-values, as they appear in the eigenmode distribution, outside the anomaly. An inspection of these figures reveals that replenishment is more efficient at lower energies. For electrons of $\gtrsim 2 \mathrm{Mev}$, replenishment by multiple Coulomb scattering east of the anomaly is negligible. Whatever high energy electron flux is found east of the anomaly in the high $B$ region, must have diffused in by means of a process other than multiple Coulomb scattering.

Dependence with B of the omnidirectional flux is very steep; some typical curves are shown in Fig. 9. Finally, the omnidirectional flux of $>300 \mathrm{kev}$ electrons at a fixed altitude of $300 \mathrm{~km}$ is plotted as a function of longitude for two $\mathrm{L}$ values in Fig. 10 .

In analyzing these results, one has to keep in mind that in the region of replenishment (high $\mathrm{B}$ ), the distribution is almost completely independent of the originally injected spectrum, and of all what happens near the equator, if multiple Coulomb scattering is the only process involved. 


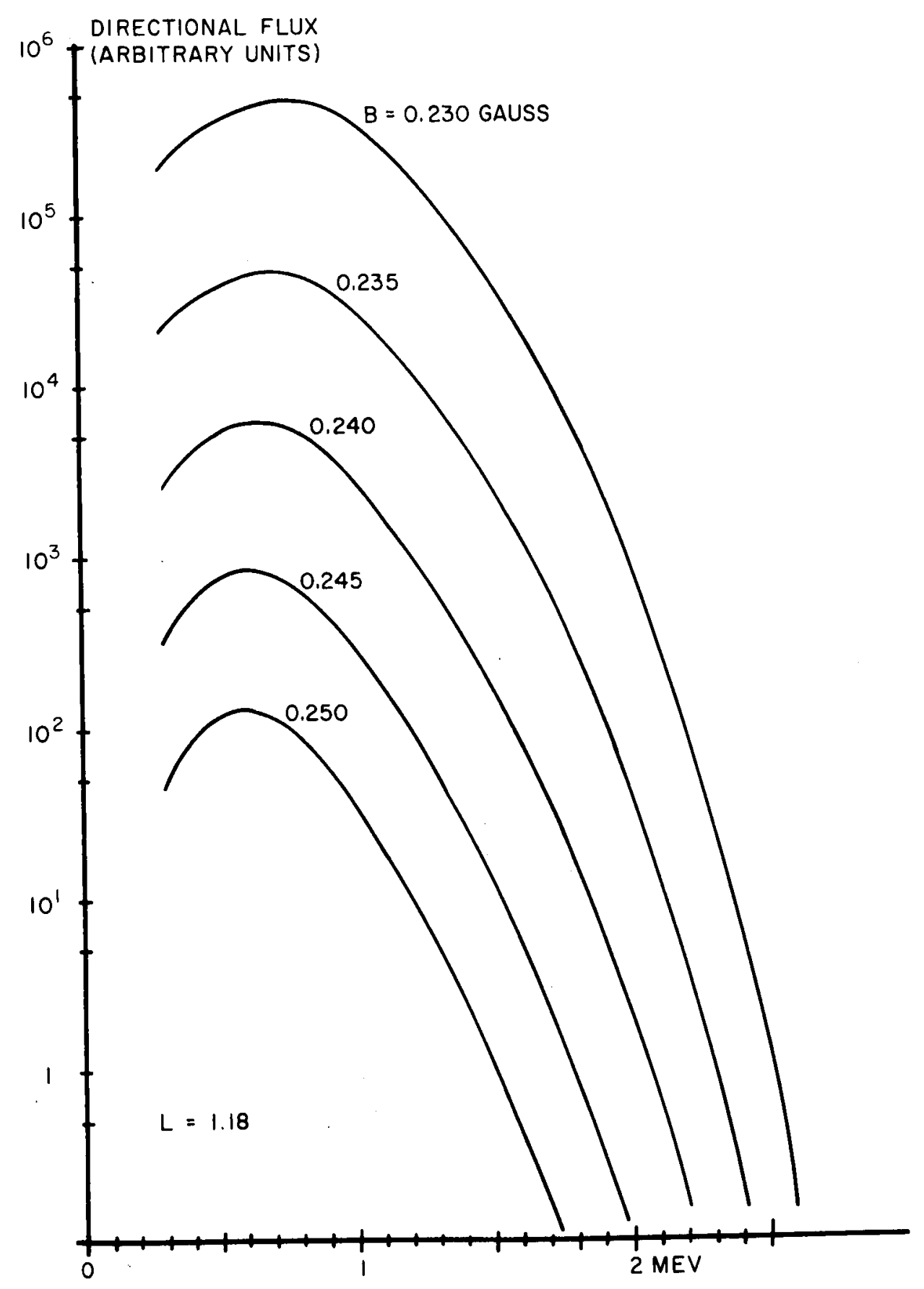

Fig. 7. Differential energy spectra of electrons mirroring at high B-values west of the anomaly, for $L=1.18$. 


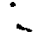

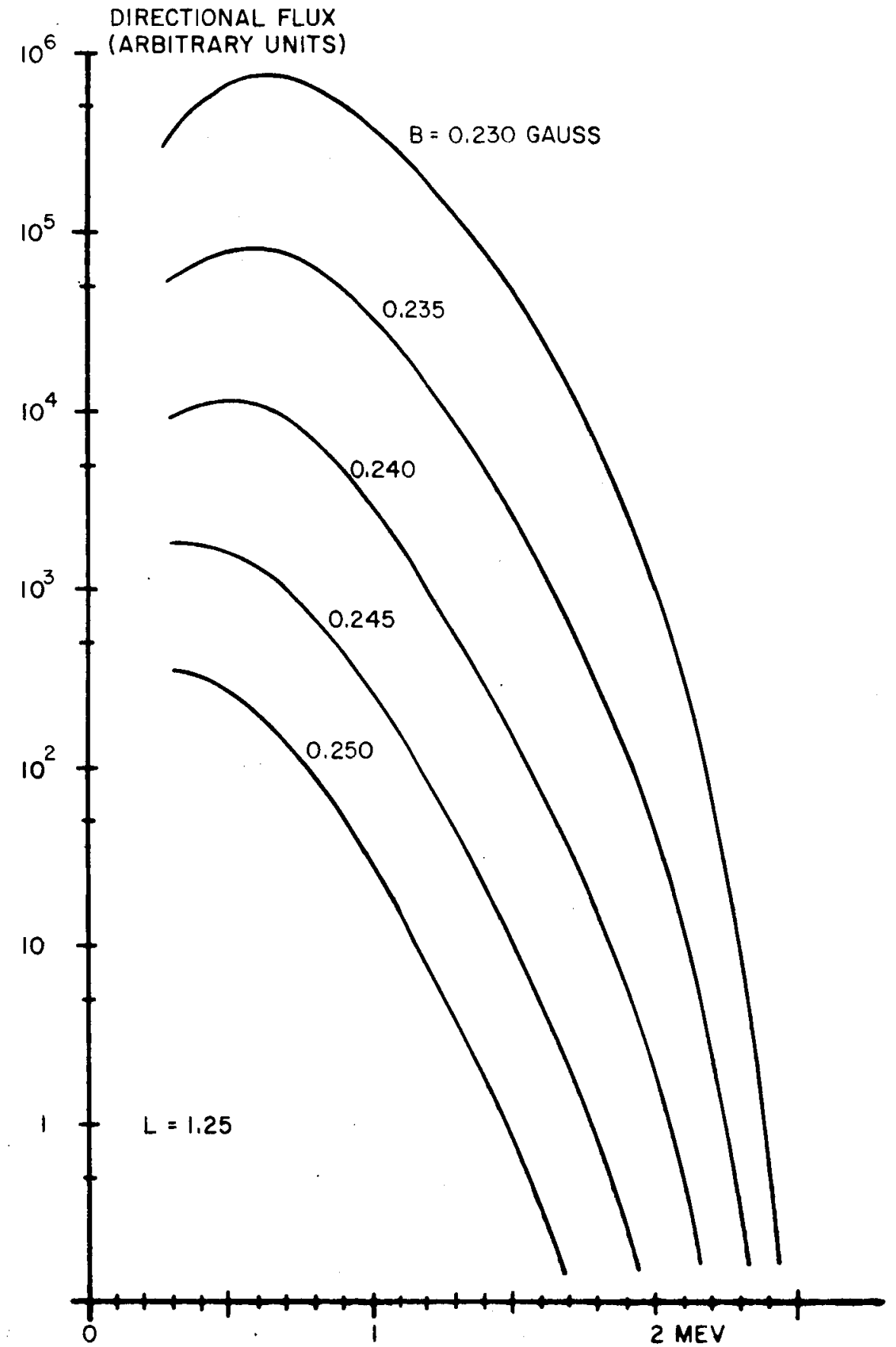

Fig. 8. Differential energy spectra of electrons mirroring at high B-values west of the anomaly, for $L=1.25$.

II -17 


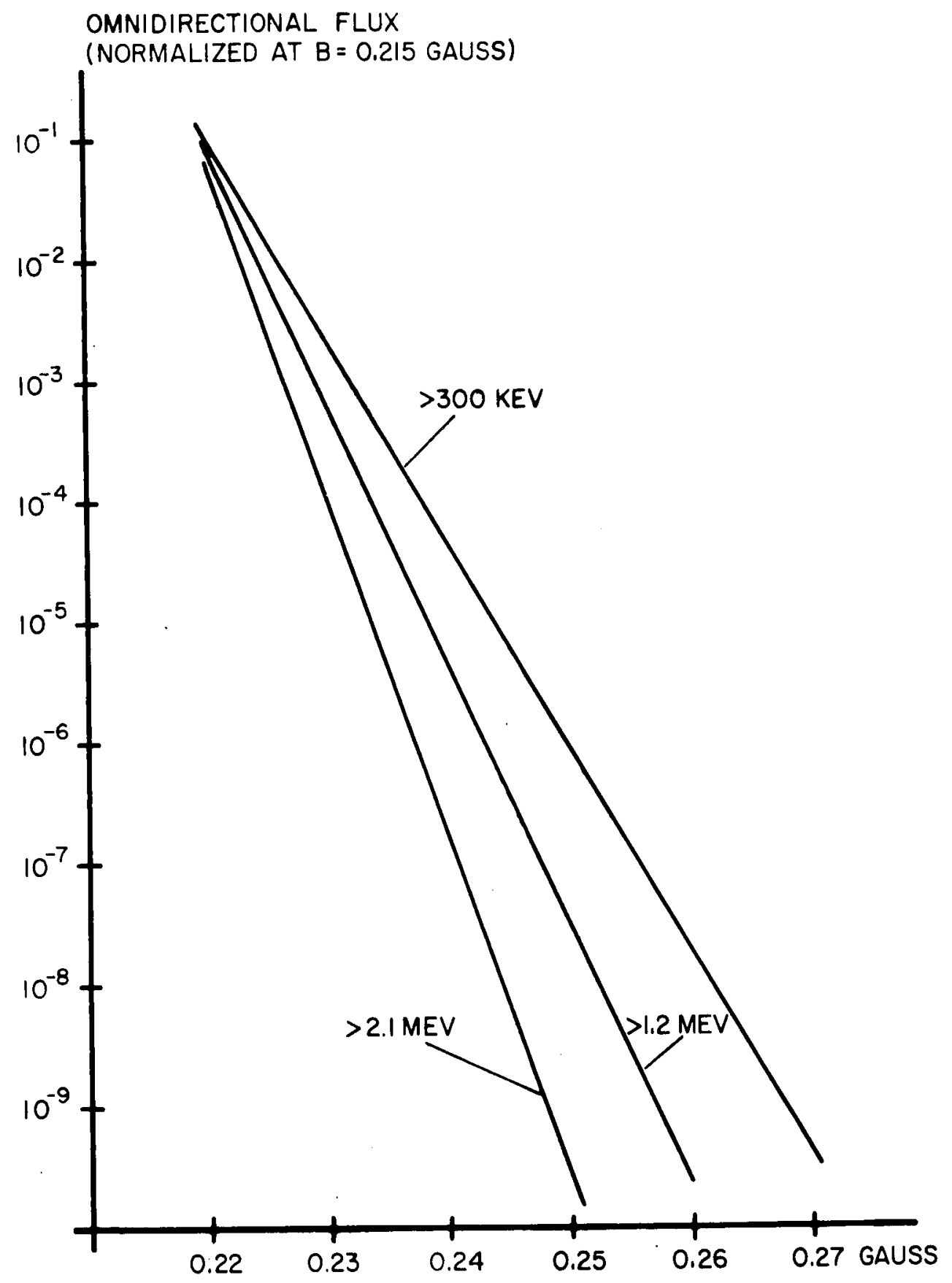

Fig. 9. B-dependence of the omnidirectional flux of electrons, for various energies. 


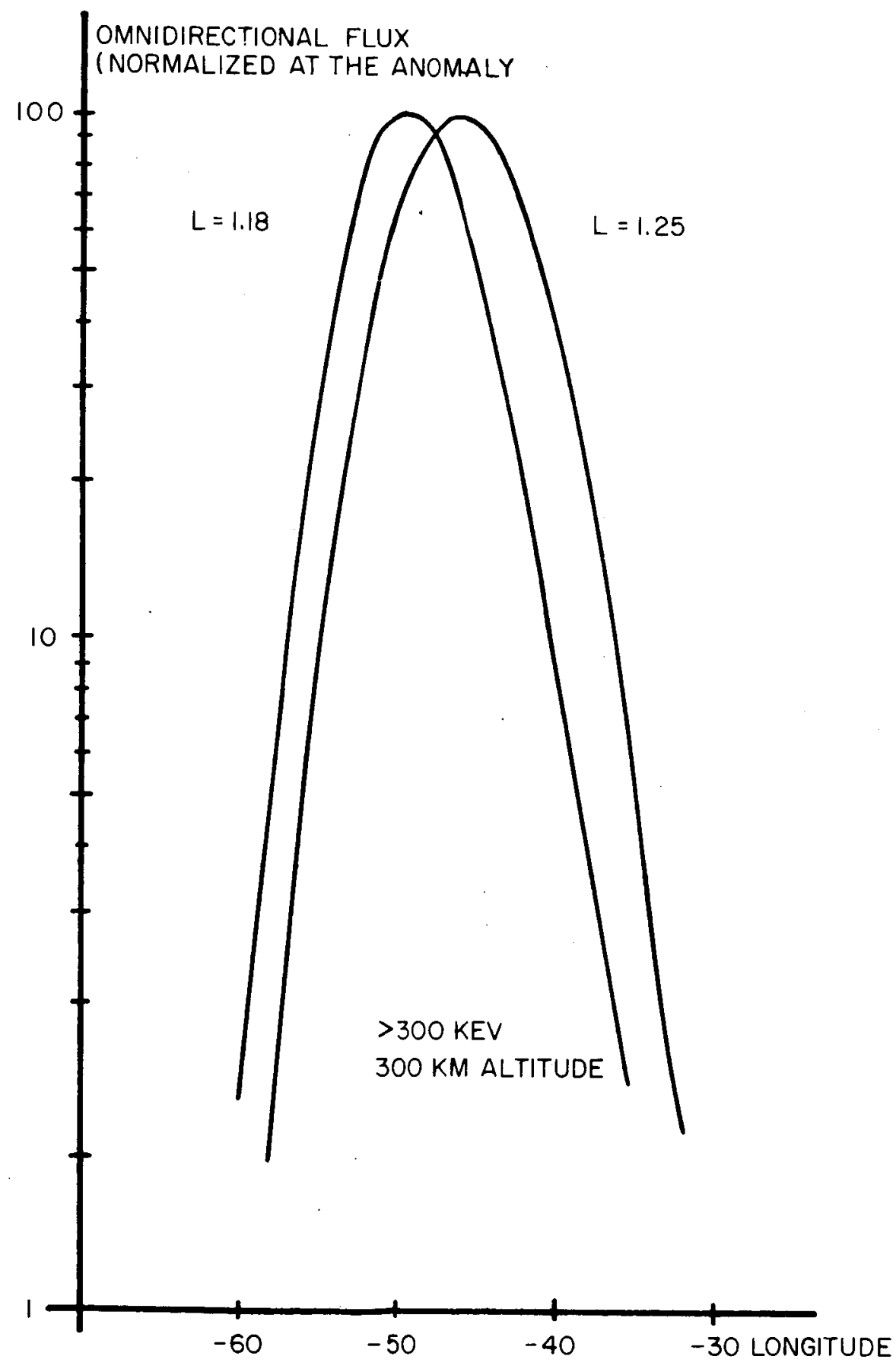

Fig. 10 Variation of the omnidirectional flux of $>300$ kev electrons al ong a constant altitude level $(300 \mathrm{~km})$, for $L=1.18$ and 1.25 .

II-19 


\section{COMPARISON WITH EXPERIMENT}

Detailed experimental data on longitude dependence of electron fluxes at low L shells are not very abundant. Only two papers deal explicitly with this subject; both describe results obtained with polar orbiting satellites. In the first one, Imhof and Smith ${ }^{12}$ present results on B and E dependence of omnidirectional electron fluxes, grouped into two longitude intervals, called "east" $\left(-30^{\circ} \leq \varphi \leq+10^{\circ}\right)$ and "west" $\left(-90^{\circ} \leq \varphi \leq-60^{\circ}\right)$ of the anomaly, for different L-shells $(\mathrm{L} \leq 1.7)$. In the second paper, Williams and Kohl ${ }^{13}$ study electron fluxes for $L \geq 1.6$ at three particular longitudes: $\varphi=0^{\circ}, 211^{\circ}$ and $282^{\circ}$.

At the present state of experimental information, only more or less qualitative comparisons with our calculations are possible. Let us consider the $\mathrm{L}=1.25$ shell, and the measured B-dependence of the omnidirectional flux (second graph in Fig. 4 of ref. 12). According to our Fig. 6, the electron distribution in the longitude interval $-30^{\circ} \leq \varphi \leq+10^{\circ}$ ("east" of the anomaly) should be almost completely "Coulomb-replenished" for the $\mathrm{L}=1.25$ shell. The computed curve for $>300 \mathrm{kev}$ electrons in Fig. 9 is in very good agreement with the experimental B-dependence, except for high B-values $(20.30$ gauss, which for field geometry reasons can have been sampled by the satellite oaly near $\left.\varphi=10^{\circ}\right)$

Imhof and Smith's results for $-90^{\circ} \leq \varphi \leq-60^{\circ}$ ("west" of the anomaly) clearly indicate the action of an additional pitch angle scattering mechaniam. The authors suggest large-angle Coulomb ecattering, by means of which equatorial particles would be scattered into high-B mirror point trajectories. We wish to point out that such a process, also atmosphere-controlled, would lead for low L shells to a replenishment process almost as longitude dependent as 
multiple scattering, being most effective in the region of the anomaly. Experimental results on electron fluxes and spectra in the region $\varphi \simeq 120^{\circ}$ are urgently needed for more conclusive studies.

We now turn to the results obtained by Williams and Kohl. Unfortunately, they refer to observations made at higher L-values than those under consideration in our theoretical and numerical analysis. One of the general conclusions of these authors, regarding $\geq 280 \mathrm{kev}$ electrons, is that electrons mirroring at B-values which remain above $350 \mathrm{~km}$ in altitude over the South Atlantic anomaly are not disturbed by the anomaly; about $90 \%$ of those that reach the $100-$ to 250 $\mathrm{Km}$ region over the anomaly are lost, and all those electrons reaching an altitude of $<100 \mathrm{~km}$ over the anomaly are lost. An inspection of the mirror point trajectories reproduced in Fig. 4 broadly confirm this.

Williams and Kohl present results picturing the "transmission" of electrons through the anomaly for different L shells. They define the "transmission" as the ratio of the counting rates obtained at $\varphi=0^{\circ}$ and $\varphi=282^{\circ}$, respectively, for a given pair of $B, L$ values. Considering the $L$ range explored, the $\varphi=0^{\circ}$ counting rates really correspond to the "inside" rather than to the east side of the anomaly. (see their Fig. 5). This means that east of their $\varphi=0^{\circ}$ point, Coulomb replenishment should still be going on for some $20^{\circ}$ to $30^{\circ}$ (remember that for higher L values, B-L rings emerge much slower out of low altitudes). True transmission factors may then be considerably higher than those quoted by Williams and Kohl. This is why a comparison of their results with our curves in Figs. 5 and 6 is not too meaningful. 
Comparison of their $\varphi=282^{\circ}$ and $\varphi=211^{\circ}$ data again clearly indicates the action of an additional, non-Coulombic pitch angle scattering mechanism, acting well east of the anomaly. The authors suggest resonant interactions with electromagnetic VLF waves. A most puzzling result is that in the high-B region, they find particle fluxes increasing by a factor 2-3 in the longitude interval between $211^{\circ}$ and $282^{\circ}$ (i.e. only $71^{\circ}$ wide), for $\mathrm{L} \simeq 2.0$. This result could never be explained by a pitch angle scattering mechanism acting with uniform efficiency over all longitudes. For the time being, it is difficult to understand why the additional scattering mechanism should be so much more effective in the 211$282^{\circ}$ longitude interval.

Electron spectra obtained in our calculations (Figs. 7,8 ) are very soft in the region of replenishment. This is in qualitative agreement with observations by Paulikas and Freden ${ }^{14}$.

The curves given in Fig. 10 are in quite poor agreement with similar plots extracted from isoflux contours shown by Mihalov et al.15 and by Vernov et al. ${ }^{16}$. The experimentally observed longitude dependence of the omnidirectional flux at a fixed altitude for a given L-shell, is much broader than the narrow "peaks" shown in Fig. 10. However, one has to keep in mind that the Mihalov et al. data were obtained for artifically injected electrons (much harder spectrum), and that Vernov et al. present GM counting rates, i.e. a superposition of electron and proton fluxes. Notice that wider peaks in a representation like Fig. 10 are equivalent to a less steep B-dependence of the flux along a field line (Fig. 9). This, again, points to the action of a non-Coulombic scattering mechanism at all longitudes. 


\section{CONCLUSIONS}

Coulomb scattering is certainly the most important pitch angle diffusion mechanism for low L-shells, but not the only one. Presently available experimental material is not detailed enough in order to reach more quantitative conclusions about the extra scattering mechanism.

It would be very useful to have more information on omni- and unidirectional electron fluxes, their energy spectra and B-dependence, at the following longitudinal positions for $L \leq 1.3$ : at the "center" of the anomaly, at $15-20^{\circ}$ east and west, respectively, and at $60-90^{\circ}$ longitude intervals, in the rest. This information should be grouped into two broad UT intervals (corresponding to day and night in the anomaly). When this information is available, it would be much simpler to "filter out" the theoretically predicted multiple Coulomb scattering contribution, and to study the behaviour of the remaining effect.

\section{ACKNOWLEDGMENTS}

We wish to express our gratitude to Mrs. Pat Comella, who collaborated efficiently in the setup of the computer program. Most of the computations were performed at the Air Force Weapons Laboratory, Kirtland AFB, N. M.; one of us (J.G.R.) expresses his gratitude to Col. R. Pennington for kind hospitality and valuable advice. 


\section{REFERENCES}

1. Cornwall, J. M. "Scattering of energetic trapped electrons by very low frequency waves," J. Geophys. Res. $\underline{69}, 1251-1258$ (1964)

2. Roberts, C. S. "Electron loss from the Van Allen zones due to pitch angle scattering by electromagnetic disturbances" Proc. NATO Advan. Study Inst., Bergen, 1965, D. Reidel, Dordrecht, Holland, to be published (1966)

3. Stix, T. A., "Theory of Plasma Waves," McGraw Hill Book Co., New York (1962)

4. Paulikas, G. A., J. B. Blake and S. C. Freden, "Precipitation of energetic electrons at middle latitudes," J. Geophys. Res. $\underline{71}$, 3165-3172 (1966)

5. Stone, E. C., "The physical significance and application of $L, B_{0}$ and $R_{0}$ to geomagnetically trapped particles, J. Geophys. Res. $\underline{68}$, 4157-4166 (1963)

6. Roederer, J. G. and J. V. Herod, "A computational model for geomagnetically trapped particle shells and kinematic parameters," Technical Report, Air Force Weapons Lab., Kirtland AFB, in press (1966)

7. Hassitt, A., "The drift velocity of trapped particles," J. Geophys Res. $\underline{70}$, $535-540(1965)$

8. Anderson, A. D., G. E. Crane, W. E. Francis, L. L. Newkirk and M. Walt, "Theoretical investigation of geomagnetically trapped electrons from high altitude nuclear explosions," Lockheed Missiles and Space Co. Report LMSC-895355 (1964) 
9. Harris, I. and W. Priester, COSPAR International Reference Atmosphere (1965)

10. Welch, J. A., R. L. Kaufmann and W. N. Hess, "Trapped electron time histories for $\mathrm{L}=1.18$ to $\mathrm{L}=1.30, " \mathrm{~J}$. Geophys. Res. $\underline{68}, 685-699$ (1963)

11. Walt, M. and L. L. Newkirk, "Interaction of trapped electrons with the atmosphere," Space Research V, North Holland Publ. Co., 458-465 (1965)

12. Imhof, W. L. and R. V. Smith, "Longitudinal variations of high energy electrons at low altitudes," J. Geophys. Res. $\underline{70}, 569-577$ (1965)

13. Williams, D. J. and J. W. Kohl, "Loss and replenishment of electrons at middle latitudes and high B values," J. Geophys. Res. $\underline{70}, 4139-4150$ (1965)

14. Paulikas, G. A. and S. C. Freden, "Precipitation of energetic electrons into the atmosphere," J. Geophys. Res. $\underline{69}, 1239-1249$ (1964)

15. Mihalov, J. D., F. S. Mozer and R. S. White, "Artifically injected electrons at low altitude," J. Geophys. Res. $\underline{69}, 4003-4013$ (1964)

16. Vernov, S. N., I. A. Savenko, P. I. Shavrin, V. E. Nesterov, N. F. Pisarenko and $\mathrm{K}$. N. Shavrina, "Investigation of the earth's radiation belts in the region of the brazilian magnetic anomaly at altitudes of $200-400 \mathrm{Km}$," Space Research V, North Holland Publ. Co., 343-359 (1965) 


\section{FIGURE CAPTIONS}

Figure 1. Angular drift velocity as a function of the longitude of the equatorial point, for particles mirroring at $B=0.18$ and 0.22 gauss, for $L=1.25$.

Figure 2. Bounce path between conjugate mirror points, of electrons mirroring at $\mathrm{B}=0.18$ and 0.22 gauss, for $\mathrm{L}=1.25$. To obtain the half-bounce period, divide by the particle velocity in earth radii/sec.

Figure 3. Sketch to illustrate the procedure of numerical integration of equation (21) (Part I).

Figure 4. Mirror point trajectories of $600 \mathrm{kev}$ electrons (average energy of electrons in the region of replenishment). Constant altitude curves are shown. Notice the narrow "window" of mirror point field intensities around $B=0.218$ gauss, west of the anomaly, which "feeds" the region of replenishment on the east side.

Figure 5. Longitude dependence of the directional flux $j$ of $300 \mathrm{kev}$ electrons mirroring at different $B$-values, for $L=1.18$.

Figure 6. Longitude dependence of the directional flux $j$ of $300 \mathrm{kev}$ electrons mirroring at different $\mathrm{B}$-values, for $\mathrm{L}=1.25$.

Figure 7. Differential energy spectra of electrons mirroring at high B-values west of the anomaly, for $\mathrm{L}=1.18$. 
Figure-8. Differential energy spectra of electrons mirroring at high B-values west of the anomaly, for $L=1.25$.

Figure 9. B-dependence of the omnidirectional flux of electrons, for various energies.

Figure 10. Variation of the omnidirectional flux of $>300 \mathrm{kev}$ electrons along a constant altitude level $(300 \mathrm{~km})$, for $\mathrm{L}=1.18$ and 1.25 . 